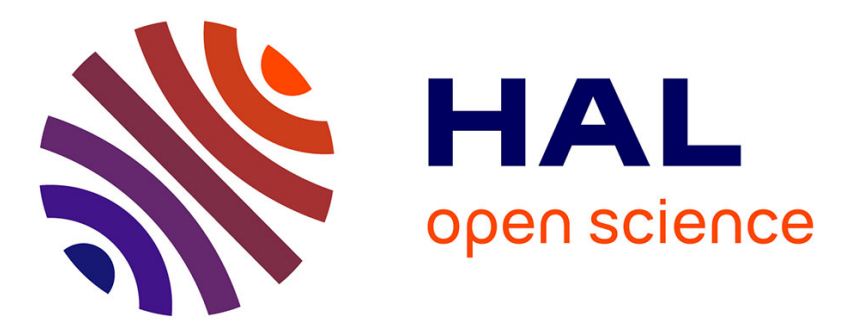

\title{
Prévention et prise en charge de l'hémorragie du post-partum au CHU Sylvanus Olympio de Lomé au Togo \\ Nadège Sitti
}

\section{To cite this version:}

Nadège Sitti. Prévention et prise en charge de l'hémorragie du post-partum au CHU Sylvanus Olympio de Lomé au Togo. Gynécologie et obstétrique. 2014. dumas-01224164

\section{HAL Id: dumas-01224164 https://dumas.ccsd.cnrs.fr/dumas-01224164}

Submitted on 4 Nov 2015

HAL is a multi-disciplinary open access archive for the deposit and dissemination of scientific research documents, whether they are published or not. The documents may come from teaching and research institutions in France or abroad, or from public or private research centers.
L'archive ouverte pluridisciplinaire HAL, est destinée au dépôt et à la diffusion de documents scientifiques de niveau recherche, publiés ou non, émanant des établissements d'enseignement et de recherche français ou étrangers, des laboratoires publics ou privés. 


\section{ACADEMIE DE PARIS \\ ECOLE DE SAGES-FEMMES - HOPITAL SAINT ANTOINE UNIVERSITE PIERRE ET MARIE CURIE - FACULTE DE MEDECINE MEMOIRE POUR LE DIPLOME D’ETAT}

\section{PREVENTION ET PRISE EN CHARGE DE \\ L'HEMORRAGIE DU POST-PARTUM AU C.H.U. SYLVANUS OLYMPIO DE LOME AU TOGO}

Directeur de mémoire : Professeur Bruno CARBONNE Année universitaire : 2013-2014

SITTI

Nadège 


\section{$\underline{\text { Remerciements }}$}

Je remercie, mon directeur de mémoire, le professeur Bruno CARBONNE, chef de service de la maternité de l'hôpital de Trousseau, pour son aide, sa disponibilité ainsi que l'intérêt qu'il a porté à cette étude. Merci à Mme Mathilde REVERT, enseignante sage-femme à l'école de Saint-Antoine, pour son encadrement et ses conseils pour la rédaction de ce mémoire.

Merci à toute l'équipe obstétricale de la maternité du CHU Sylvanus Olympio pour leur accueil chaleureux et leur disponibilité, en particulier le professeur Koffi APKADZA de m'avoir permise de réaliser cette étude au sein de son service.

Je remercie tous ceux qui m'ont toujours soutenu : mes parents, mes frères, toute ma famille en France et au Togo ainsi que mes amies, en particulier à Dylène DESIR et à Marielle TEGUH (ma princesse des eaux).

Je tiens tout particulièrement à remercier Camille VERHAEGHE pour sa grande patience, son soutien, et ses encouragements dans les moments difficiles.

Enfin, je dédie tout spécialement ce mémoire à ma chère et tendre maman. 


\section{SOMMAIRE}

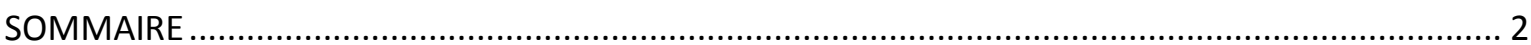

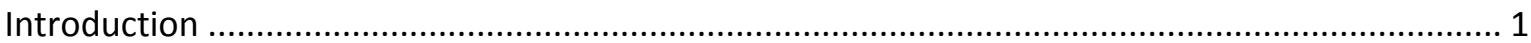

Première partie: contexte général

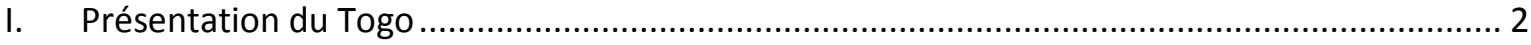

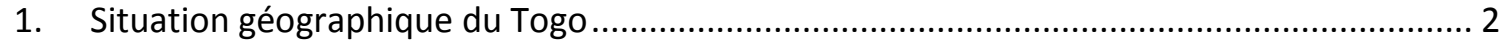

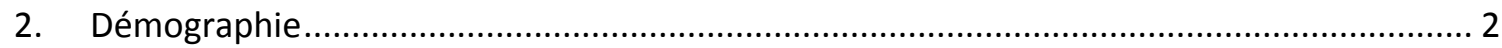

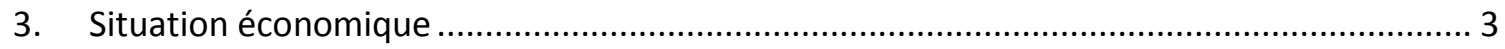

4. Schéma d'organisation sanitaire du Togo en 2009 ............................................................ 3

II. Hémorragie du post-partum immédiat (HPPI) ........................................................................ 4

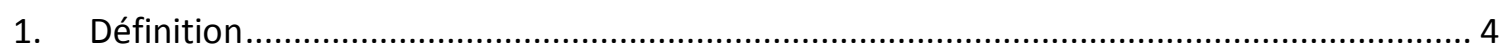

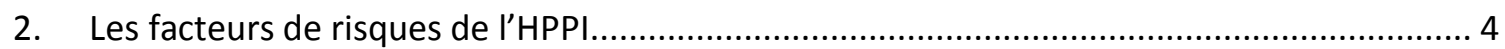

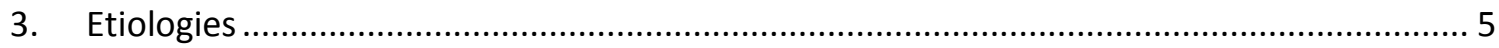

4. Gestion Active de la Troisième Phase de l'Accouchement ................................................. 6

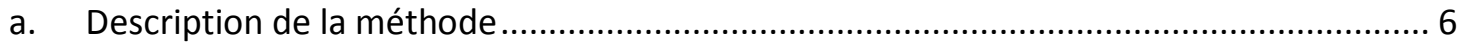

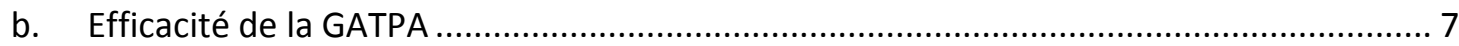

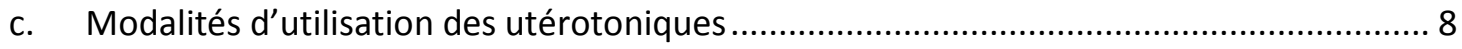

III. Etat des lieux de la prévention et de la prise en charge de l'HPPI...................................... 10

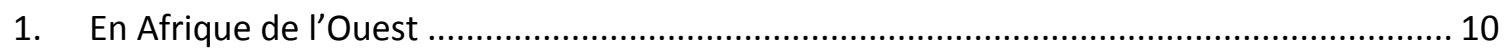

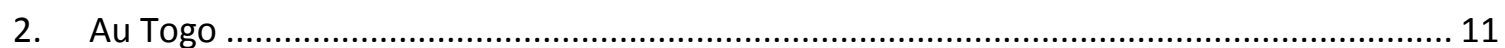

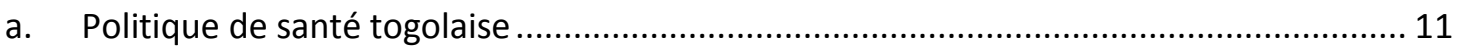

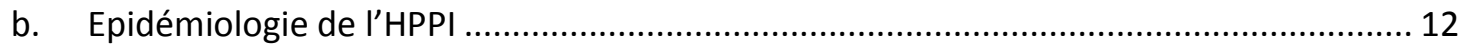

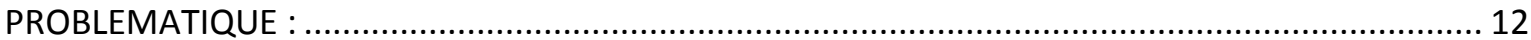

Deuxième partie : Méthodologie

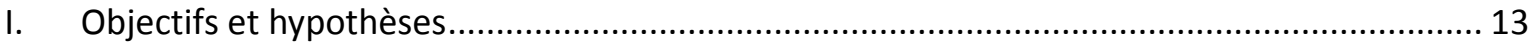

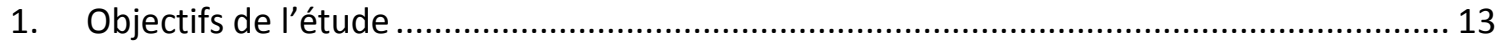

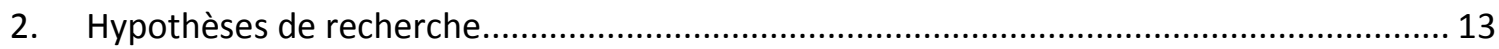

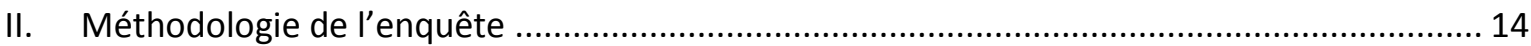

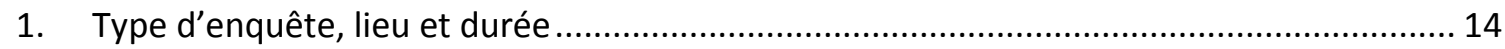

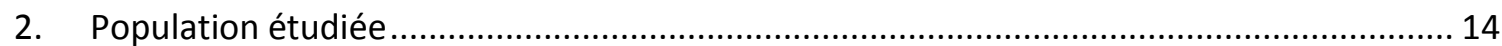


3. Variables analysées dans l'étude descriptive de la prévention et de la prise en charge de

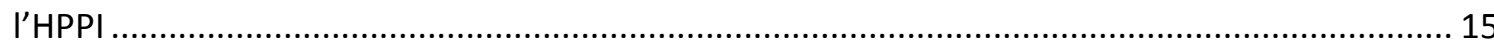

a. Caractéristiques générales et obstétricales ............................................................... 15

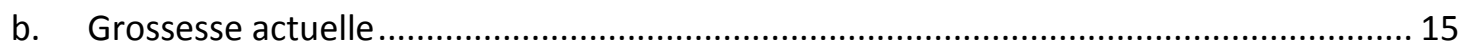

c. Déroulement du travail et de l'accouchement .............................................................. 15

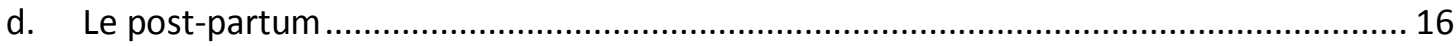

e. La prise en charge de l'hémorragie du Post-partum Immédiat ......................................... 16

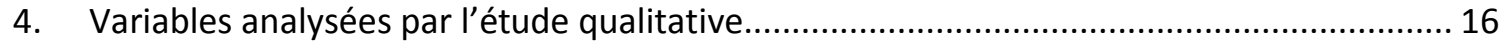

Troisième partie : Résultats

I. Description du système de soins au Togo et du circuit du patient en maternité ..................... 17

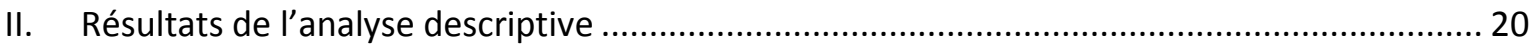

1. Prévention de l'hémorragie du post-partum immédiat (HPPI) ........................................... 20

a. Caractéristiques des patientes admises en travail.............................................................. 20

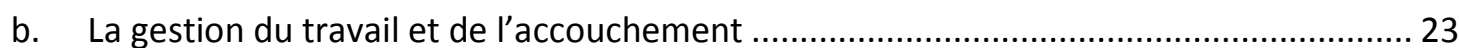

c. La délivrance : troisième phase de l'accouchement ........................................................... 24

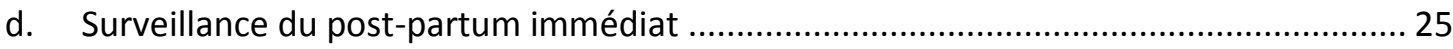

2. Prise en charge de l'hémorragie du post-partum immédiat.............................................. 27

a. Caractéristiques et prise en charge des patientes ayant accouchées au CHU-SO............2 27

b. Caractéristiques et prise en charge des patientes transférées pour HPPI........................ 30

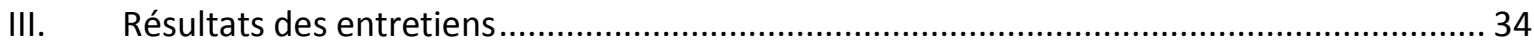

Quatrième partie : Discussion

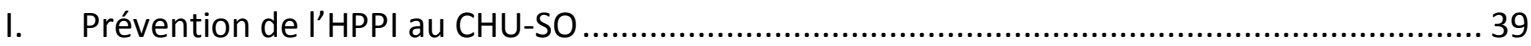

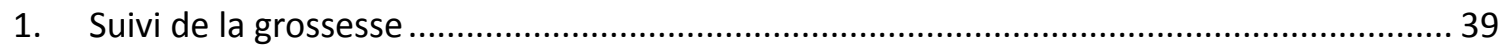

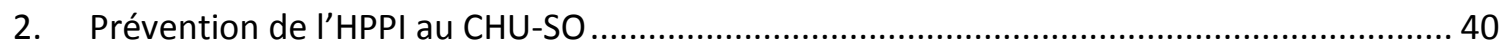

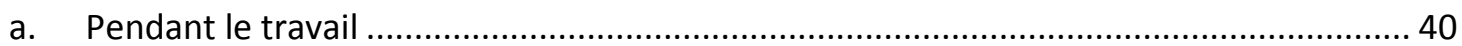

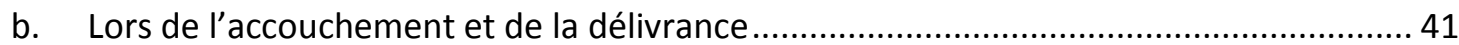

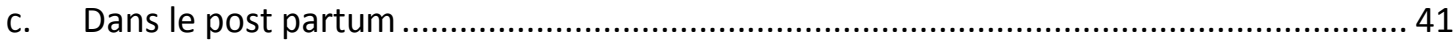

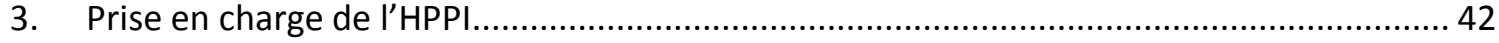

a. Les connaissances des professionnels ............................................................................ 42

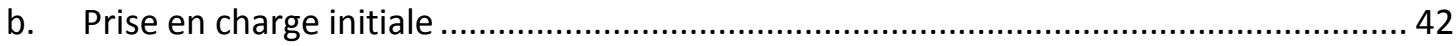

c. Cas particuliers des patientes transférées : rôle des centres de soins primaires ............ 43

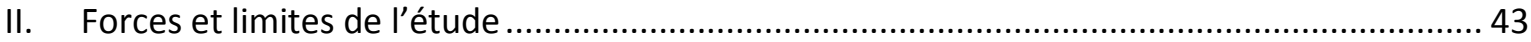

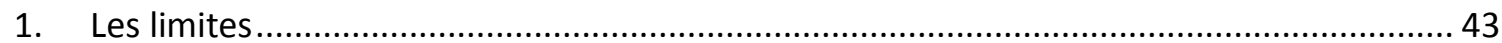




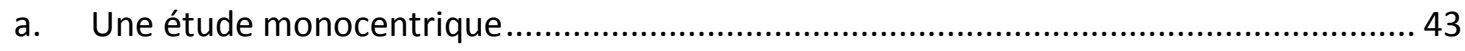

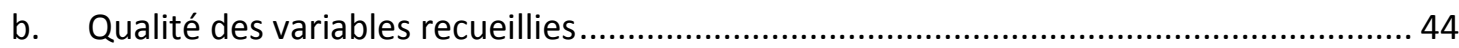

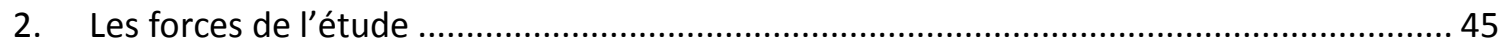

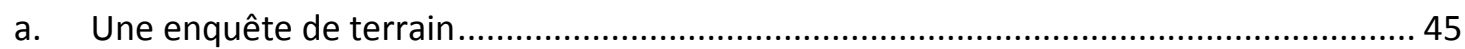

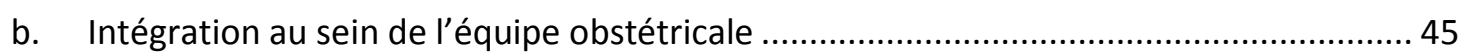

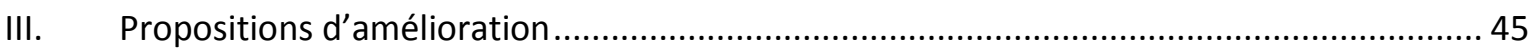

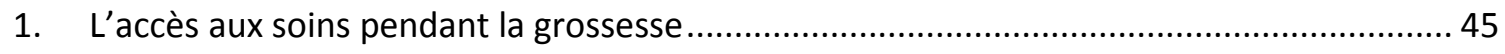

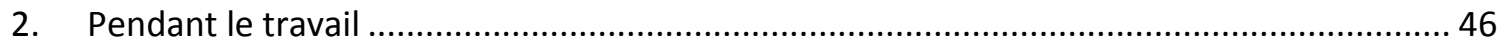

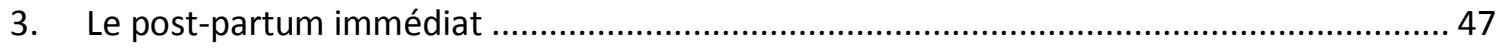

a. La prévention de l'HPPI ................................................................................................... 47

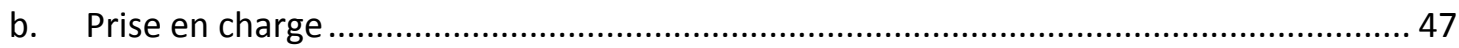

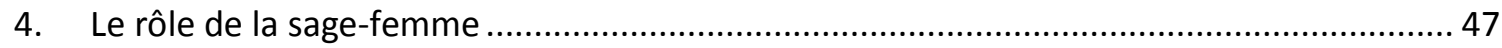

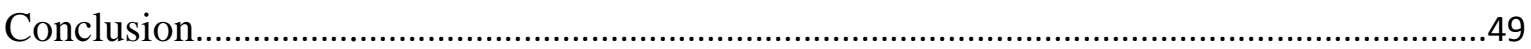

Annexes

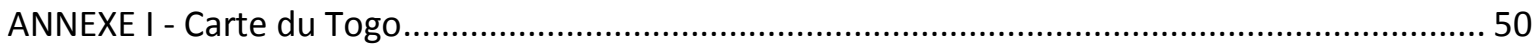

ANNEXE II- Gestion active de la troisième phase de l'accouchement ............................................. 51

ANNEXE III- Grille de remboursement des soins par I'INAM ........................................................... 52

ANNEXE IV - RPC des soins obstétricaux d'urgence en Afrique .................................................... 53

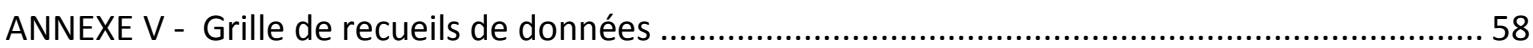

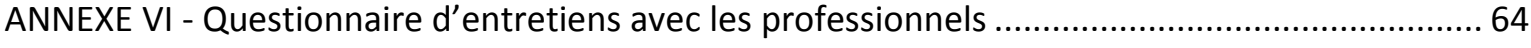

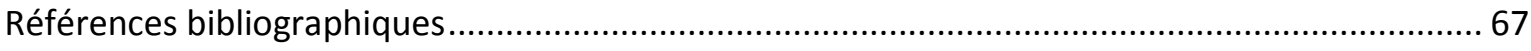

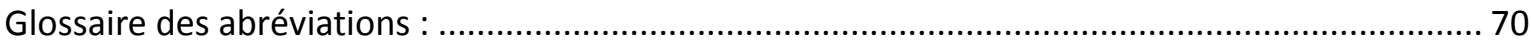




\section{Liste des tableaux et figures}

\section{Figures}

Figure 1 : Mortalité maternelle dans le monde en 2008

Figure 2 : Modalités de la délivrance.

\section{Graphiques}

Graphique 1 : Motifs d'admission des patientes référées pendant le travail..................................21

Graphique 2 : Patientes ayant reçu du Misoprostol par voie rectale................................................26

\section{$\underline{\text { Tableaux }}$}

Tableau 1 : Caractéristiques des patientes admises en travail......................................................20

Tableau 2 : Etude des facteurs de risques d'HPPI préexistant au travail.........................................22

Tableau 3 : Modalités du déroulement du travail..........................................................................23

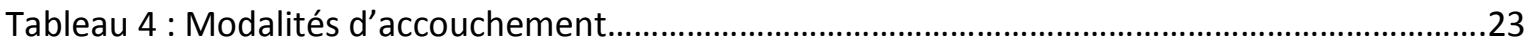

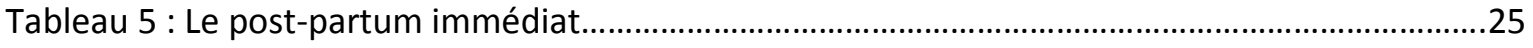

Tableau 6 : Patientes ayant fait une HPPI après un accouchement au CHU-SO.............................28

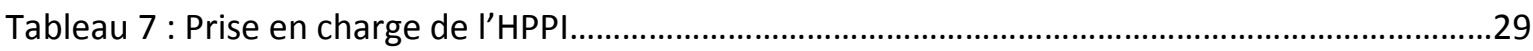

Tableau 8 : Caractéristiques des patientes transférées pour HPPI..............................................30

Tableau 9 : « Histoire » des patientes transférées ..........................................................................31

Tableau 10 : Prise en charge des patientes transférées..................................................................32

Tableau 11 : Transfusion sanguine en fonction du délai de transfert des patientes.......................33

Tableau 12 : Résultats de l'entretien avec les professionnels ............................................... 36 


\section{Introduction}

Le parcours personnel et professionnel souhaité, a orienté le choix du travail de recherche du mémoire qui sera présenté au diplôme d'état de sage-femme 2014. La réflexion s'est engagée dès le début de la recherche bibliographique sur cette thématique: "Dans le monde, toutes les $8 h$, on enterre une mère suite à une complication liée à la grossesse, à l'accouchement ou aux suites de couches ${ }^{1} \gg$. Pour l'Institut National français des études démographiques(INED) dans plus de $20 \%$ des cas, l'hémorragie est la cause des décès maternels(1).

En 2010, on estime à 287000 le nombre total de femmes décédées dans le monde suite à une complication due à la grossesse. $85 \%$ de ces décès maternels ont lieu dans les pays en développement, dont les trois cinquièmes surviennent en Afrique subsaharienne et pendant la période gravido-puerpérale. Le choix du travail de mémoire a été motivé par ce taux important de mortalité maternelle. En effet, dans certains pays africains on compte près de 1000 décès maternels pour 100000 naissances vivantes (soit 1\%) (2). Le sujet de ce travail est la prévention et la Prise en charge (PEC) de l'Hémorragie du post-partum immédiat (HPPI) dans un pays en développement.

La présente étude a fait l'objet d'une investigation sur le terrain dans le cadre du stage optionnel réalisé en troisième année d'études de sages-femmes; ceci pendant l'été 2013 au Centre Hospitalier Universitaire Sylvanus Olympio (CHU-SO) de Lomé, au Togo, mon pays natal.

Ce stage, avait plusieurs objectifs : le premier était la réalisation d'une étude descriptive nécessaire au travail du mémoire de fin d'études ; les objectifs secondaires étaient la réalisation d'un travail de recherche s'inscrivant dans le champ de la périnatalité, et d'avoir un regard objectif sur la prise en charge de la santé génésique des femmes ouest-africaines, plus particulièrement des femmes togolaises ; enfin, ce stage de trois semaines, auprès de professionnels de santé démunis sur le plan matériel et de femmes capables d'humanité et de générosité, malgré leurs conditions de vie m'a conforté dans mon projet professionnel dans le cadre de l'humanitaire.

Dans une première partie sera décrit le contexte général du travail, c'est-à-dire une description de la prévention et de PEC de l'HPPI selon l'Organisation Mondiale de la Santé ainsi que l'exposé du contexte propre à l'Afrique de l'ouest. La méthodologie de l'enquête, sera détaillée dans la partie méthodologie. Les résultats seront exposés dans une troisième partie et discutés en quatrième partie.

1 Folly D. H. Lutte contre la mortalité maternelle : le Togo en campagne contre un drame persistant. Septembre 2010 


\section{Première partie : contexte général}

\section{Présentation du Togo}

\section{Situation géographique du Togo}

Situé en Afrique de l'Ouest, le Togo est l'un des plus petits états africains, s'étirant sur 700km du Nord au Sud avec une largeur n'excédant pas $100 \mathrm{~km}$. Ses pays limitrophes sont le Burkina-Faso (au nord), le Bénin (à l'Est) et le Ghana (à l'ouest). Il est limité au sud par le Golfe de Guinée.

Le Togo est divisé en cinq régions administratives, elles-mêmes découpées en 30 préfectures s'étendant sur une superficie totale de $56785 \mathrm{~km}^{2}(4)$ (ANNEXE I).

\section{Démographie}

En 2013, la population togolaise fut estimée à 7154237 d'habitants avec un taux d'accroissement naturel de 2,74\% et une densité de $126 \mathrm{hab}$. $/ \mathrm{km}^{2}$. Le taux de natalité est de 35,26 pour 1000 tandis que celui de mortalité est de 7,78 pour 1000. L'espérance de vie à la naissance est de 60 ans chez les femmes et 55 ans chez les hommes.

La population togolaise est une population très jeune. En effet, près de la moitié de la population a moins de 18 ans. 


\section{Situation économique}

Membre de la CEDEAO (Communauté Economique des Etats de l'Afrique de l'Ouest), la monnaie togolaise est le franc $\mathrm{CFA}^{2}$.

Avec un PIB par habitant de 574\$, l'économie du Togo correspond à celui d'un pays en voie de développement. Mais malgré un taux de croissance estimé à 5\% en 2012, le Togo demeure un des pays les plus pauvres, du fait notamment de la gestion des richesses, et de la croissance démographique. En effet, 58,7\% de la population vivent sous le seuil de la pauvreté.

\section{Schéma d'organisation sanitaire du Togo en 2009}

1. L'organisation du système de soins togolais est de type pyramidal à 3 niveaux : (5) Le sommet de la pyramide constitue le niveau central ou national. On y retrouve le cabinet du ministre de la santé, la direction générale de la santé, 5 directions centrales, 15 divisions et 39 services centraux, et différents programmes. Les structures de soins sont représentées par les Centres Hospitaliers Universitaires (CHU) (3 au total dont 2 à Lomé) qui constituent le $3^{\text {ème }}$ niveau de référence.

Le rôle de ce niveau est de définir les grandes orientations de la politique sanitaire du pays.

2. Le niveau intermédiaire ou régional se situe au milieu de la pyramide. Il est représenté par 6 régions sanitaires : Lomé-Commune, Maritime, Plateaux, Centrale, Kara, Savanes et également les 6 Centres Hospitaliers Régionaux (CHR) constituant le deuxième niveau de référence du système de soins. Ces structures sont chargées de l'appui et du suivi de la mise en œuvre en régions de la politique de santé et des directives nationales.

3. La base de la pyramide constitue le niveau périphérique ou opérationnel.

Il est composé de 30 directions préfectorales de santé et représenté par les 35 hôpitaux de district, 7 hôpitaux confessionnels, 85 centres médico- sociaux (CMS), 10 centres de santé, 405 unités de soins périphériques, 41 centres de protection maternelle et infantile, plusieurs cabinets et cliniques privées.

\footnotetext{
2 1euro $=655$ FCFA
} 


\section{Hémorragie du post-partum immédiat (HPPI)}

\section{Définition}

Selon l'OMS, l'hémorragie du post-partum immédiat (HPPI) se définit par une perte sanguine supérieure à 500 millilitres $(\mathrm{ml})$ dans le cas d'un accouchement voie basse et d'une perte de 1000 $\mathrm{ml}$ dans le cas d'une césarienne, dans les 24 heures suivant la naissance. Une perte sanguine supérieure à $1000 \mathrm{ml}$ dans le cas d'un accouchement normal est définie comme étant une hémorragie sévère.

\section{Les facteurs de risques de l'HPPI}

Les études menées dans le cas de l'HPPI, ont permis de mettre en évidence de nombreux facteurs de risques $(6,7)$.

On distinguera, les facteurs de risques identifiables pendant la grossesse et les facteurs de risques liés à l'accouchement.

- Facteurs de risques identifiables pendant la grossesse :

- âge maternel : un âge maternel $\geq 35$ ans augmente indirectement le risque d'HPPI en augmentant les risques de complications obstétricales et non les étiologies directes de l'HPPI telles que l'atonie ou la rétention placentaire. (8)

- un bas niveau socio-éducatif, notamment dans les pays en voie de développement

- un antécédent d'hémorragie du post-partum

- l'obésité

- une surdistension utérine (due à une macrosomie, une grossesse multiple, un hydramnios ou encore à la multiparité)

- $\quad$ un placenta prævia (9)

- $\quad$ un hématome rétro placentaire (HRP)

- un utérus cicatriciel

- une grossesse non suivie

- une pré-éclampsie

- une anémie pendant la grossesse (occasionne une moindre tolérance maternelle)

- des troubles de la coagulation 
- La gestion du travail peut aussi constituer un risque d'HPPI :

- le lieu de l'accouchement (présence ou non d'une équipe obstétrico-anesthésiste sur place)

- un déclenchement artificiel du travail

- l'usage excessif et inadapté d'ocytocine

- un travail long

- un travail dystocique

- un travail rapide

- une infection ovulaire

- une césarienne en urgence

- une extraction instrumentale associée à une épisiotomie

- l'utilisation d'anesthésiques halogénés qui ont un effet utéro-relaxant

Ces facteurs de risque doivent toujours être pris en compte même si les conclusions de ces études ne nous indiquent pas quelles patientes doivent faire l'objet de mesures spécifique de prévention pendant la grossesse. En effet, l'HPPI survient deux fois sur trois en l'absence de facteur de risque. Ainsi donc, il est nécessaire de mettre en place des moyens de prévention pour toutes les parturientes quel que soit le mode et le lieu d'accouchement.

\section{Etiologies}

Plusieurs étiologies ont été mises en évidence dans les études.

- La plus fréquente est l'atonie utérine. Elle se définit par l'absence de contractions utérines et représente plus de la moitié des hémorragies du postpartum.

- Ensuite, on retrouve la rétention placentaire, deuxième cause d'HPPI. Cette rétention peut être partielle ou complète et peut concerner tant les membranes placentaires que les cotylédons.

- Les lésions du tractus génital (déchirure cervicale, déchirure du vagin, de la vulve ou du périnée) constituent aussi une cause d'hémorragie du post-partum.

- Enfin on retrouve les troubles de la coagulation et plus rarement l'inversion utérine. 


\section{Gestion Active de la Troisième Phase de l'Accouchement}

\section{a. Description de la méthode}

En 2003, la Fédération internationale des gynécologues-obstétriciens (FIGO) et la Confédération internationale des sages-femmes (ICM) publient conjointement un rapport sur la nécessité de la prise en charge de la délivrance : la «Gestion active de la troisième phase de l'accouchement » (GATPA). (10)

«La prise en charge active du troisième stade du travail devrait être offerte à toutes les femmes puisqu'elle réduit la fréquence de l'hémorragie post-partum liée à l'atonie utérine »

(Annexe II)

La GATPA consiste en :

\section{Injection d'un utérotonique :}

L'injection de l'utérotonique doit se faire dans la minute suivant l'expulsion fœtale (et seulement après vérification par palpation abdominale qu'il n'y a pas un autre fœtus).

L'utérotonique de choix est l'ocytocine de synthèse (Syntocinon®). Dix unités internationales (UI) d'ocytocine doivent être injectées en intramusculaire.

Dans les régions où l'ocytocine n'est pas disponible, d'autres utérotoniques peuvent être utilisés. C'est le cas du métylergométrine qui est un dérivé de l'ergot de seigle. Cette substance n'est plus utilisée dans les pays développés du fait des effets secondaires importants (troubles gastrointestinaux, vasculaires cardiaques potentiellement graves).

Enfin, le misoprostol (Cytotec $®)$, peut aussi être utilisé en prévention de l'HPPI. La patiente doit prendre $600 \mu \mathrm{g}$ de misoprostol par voie orale.

L'utilisation d'utérotonique vise à augmenter les contactions utérines afin de faciliter le décollement placentaire.

\section{Traction contrôlée du cordon}

La traction contrôlée doit se faire de manière précise et est secondaire au clampage du cordon (après arrêt des battements cardiaques fœtal dans le cordon soit environ deux minutes après la naissance). 
Le professionnel doit exercer à la fois une traction douce du cordon vers le bas, ainsi qu'une contre-pression sus-pubienne qui vise à stabiliser l'utérus.

Des efforts expulsifs de la parturiente peuvent faciliter le geste de l'accoucheur. Ces efforts de poussée maternelle et la traction contrôlée du cordon doivent être contemporains d'une contraction utérine.

Lors de l'expulsion, la galette placentaire doit être tenue à deux mains par le professionnel ; afin de tourner délicatement l'organe sur lui-même et assurer ainsi l'expulsion des membranes fœetales dans leur totalité.

Un examen du placenta doit être fait dans le but de s'assurer que la délivrance est complète.

\section{Massage utérin}

Le massage utérin vise à stimuler l'utérus pour s'assurer de sa contractilité.

Selon la FIGO, ce massage doit être répété toutes les quinze minutes pendant les deux premières heures suivant l'accouchement (11).

Dans un rapport publié en 2010, l'OMS notifie une diminution significative des pertes sanguines dans les 30 minutes suivant l'expulsion en cas de massage utérin. Dans le but de développer la GATPA dans les pays les plus touchés par la mortalité maternelle due à l'HPPI, l'Agence américaine pour le développement international (USAID) en association avec la FIGO et l'ICM ont mis en place l'Initiative de prévention de l'hémorragie du post-partum (POPPHI) en 2004 (12).

\section{b. Efficacité de la GATPA}

Une méta-analyse publiée dans la Cochrane Library en 2000 (13) reprend cinq études comparatives menées entre 1988 et 1998 sur l'expectative versus la gestion active de la délivrance. Au total, 6500 femmes ont été incluses dans cette étude. Quatre des cinq études étaient de bonne qualité.

Le résultat principal montre que la prise en charge active de la délivrance permet une diminution des pertes sanguines suite à l'accouchement (différence moyenne pondérée $=-79,33$ millilitres [intervalle de confiance à $95 \%$ (IC95) : de -94,29 à -64,37]), une diminution du risque d'hémorragie de la délivrance, (risque relatif $(\mathrm{RR})=0,38$ [IC95 : 0,32-0,46]), ainsi qu'une diminution du délai d'expulsion du placenta (de 10 minutes en moyenne). Par contre cette étude met en évidence que la prise en charge active est associée à une augmentation des risques de nausées, de vomissements et d'hypertension artérielle dus notamment à l'utilisation de l'ergométrine. 


\section{c. Modalités d'utilisation des utérotoniques}

Dans le cas de la GATPA, les utérotoniques les plus couramment utilisés sont l'ocytocine de synthèse (ou Syntocinon $®$ ) et le misoprostol (Cytotec $®)$.

Comme vu précédemment, l'ocytocine représente l'utérotonique de choix dans le cadre de la GATPA, d'une part du fait de son délai d'action rapide (2-3minutes), et surtout de ses effets secondaires quasi-inexistants. Ainsi donc, elle peut être administrée à toutes les parturientes. Mais l'ocytocine de synthèse présente un inconvénient qui pourrait limiter son utilisation dans les pays tropicaux en développement : les ampoules d'ocytocine doivent être conservées au réfrigérateur à une température comprise entre $+2^{\circ} \mathrm{C}$ et $+8^{\circ} \mathrm{C}(14)$.

De plus, l'utilisation de l'ocytocine nécessite non seulement la compétence des soignants à réaliser des injections mais aussi la disponibilité de matériels stériles.

Le misoprostol est le deuxième utérotonique pouvant être utilisé. Il s'agit d'un analogue de la prostaglandine E1. Il est conservé à température ambiante. L'administration se fait par voie orale, sublinguale ou rectale et ne nécessite donc aucune formation particulière des accoucheurs.(15)

De nombreux essais randomisés montrent, une diminution significative du taux d'HPP, que ce soit des HPP modérées ou des HPP sévères, lors de l'utilisation préventive du misoprostol. L'essai de Mobeen et al. réalisé au Pakistan en 2011 démontre l'efficacité de l'utilisation de $600 \mu \mathrm{g}$ de misoprostol par voie orale lors de la troisième phase de l'accouchement. Le taux d'HPP était de $16,5 \%$ avec le misoprostol contre $22 \%$ avec un placebo (risque relatif $=0,76[$ IC95: $0,59-0,97]$ ) (16).

Les effets secondaires notables sont les frissons avec parfois une hyperthermie. Dans les études on note dans de rares cas, des épisodes de nausées et/ou de vomissements et de diarrhées.

L'érgométrine est quant à elle surtout efficace dans le traitement de l'HPPI. Mais les études ont démontré un grand nombre d'effets secondaires tels que l'hypertension artérielle, céphalées, nausées, vomissements. Son utilisation est donc contre-indiquée chez les patientes hypertendues, ayant une pré-éclampsie, ou des pathologies cardio-vasculaires. De plus son administration se fait par voie parentérale et la molécule est très instable à la chaleur et à la lumière.

En 2001, en comparant l'administration par voie rectale de $800 \mu \mathrm{g}$ de misoprostol versus une association d'ergométrine et d'ocytocine par voie intraveineuse, Lokugamage et al. ont démontré que le misoprostol était bien plus efficace que l'association Syntocinon®/ergométrine dans le traitement de l'HPPI (17).

En 2007, Gülmezoglu a démontré la supériorité de l'ocytocine sur le misoprostol dans la prévention de l'HPPI (3,3\% contre 2,4\%). L'efficacité de l'ocytocine a aussi été démontrée dans le traitement de l'HPP (18). 
Dans le cas de la GATPA, la FIGO et l'OMS recommandent l'injection en intramusculaire de 10UI de Syntocinon®, car il a été prouvé que cinq UI de Syntocinon ${ }^{\circledR}$ en monothérapie par voie intramusculaire sont moins efficaces que l'association de cinq UI Syntocinon ${ }^{\circledR}$ avec 0,5 milligrammes (mg) d'ergométrine (19). Mais du fait des effets secondaires et des contre-indications de l'ergométrine vus précédemment, les organismes internationaux de santé ont jugé préférable de doubler la dose d'ocytocine et de supprimer l'ergométrine afin d'éliminer les effets secondaires tout en gardant la même efficacité d'action.

Au total, l'ocytocine de synthèse est l'utérotonique de choix dans la prévention et la prise en charge thérapeutique de l'hémorragie du post-partum due à une atonie utérine. Mais dans les régions à faibles ressources médicales, où l'ocytocine n'est pas disponible, le misoprostol reste une alternative acceptable. En 2011, l'OMS, inscrit le misoprostol sur la liste des médicaments essentiels à la prévention de l'HPP. 


\section{Etat des lieux de la prévention et de la prise en charge de $\underline{\text { l'HPPI }}$}

\section{En Afrique de 1'Ouest}

L'hémorragie du post-partum immédiat (HPPI) constitue la première cause de mortalité maternelle dans le monde. En 2000, selon l'OMS, la prévalence de l'HPPI en Afrique de l'Ouest était de 1,9\% (figure 1).

La prévention primaire de l'hémorragie du post-partum repose sur un suivi régulier de la grossesse. L'OMS recommande au minimum quatre consultations prénatales (CPN) durant la grossesse (20).

Une étude réalisée au Sénégal et au Bénin montre que les femmes béninoises réalisent en moyenne six CPN tandis que les sénégalaises ne consultent que 2 à 3 fois durant leur grossesse. De plus, au Sénégal, nombre de femmes ne sont suivies pendant leur grossesse que par des accoucheurs traditionnels (21).

Au Congo, une étude prospective réalisée entre 2006 et 2008, à la maternité de l'hôpital de Brazzaville met en évidence que 3,7\% des patientes accouchent en dehors d'une structure sanitaire. Parmi les raisons évoquées par les patientes, on retrouve l'ignorance des signes cliniques de début de travail (61\%) puis l'attente de l'entrée en travail avant de se présenter à l'hôpital (46\%), alors même que plus de la moitié de ces parturientes habitaient à proximité d'une structure sanitaire. Le motif d'admission après l'accouchement était une hémorragie dans $58 \%$ des cas (22). Ces chiffres montrent l'intérêt d'un suivi obstétrical régulier dans la prévention de l'HPP.

Dans une étude réalisée en 1994 au CHU de Brazzaville au Congo (23), l'étiologie principale était l'atonie utérine (42\%), suivie de troubles de la coagulation puis enfin la rétention placentaire, responsable de $25 \%$ des hémorragies du post-partum. Le traitement médical reposait sur l'utilisation d'ocytocine et de méthylergométrine. Les gestes obstétricaux ont été la délivrance artificielle, la révision utérine, le massage utérin, la traction sur le col et enfin l'hystérectomie d'hémostase dans les cas les plus sévères. Le taux de mortalité fut de 39\%, principalement dû à un trouble de la coagulation. Le manque de produits sanguins était l'une des causes de ce fort taux de mortalité. 
Au Ghana, en conformité avec les recommandations de la FIGO et de l'ICM, le ministère de la santé ainsi que les associations nationales de sages-femmes et de gynécologues-obstétriciens recommandent l'utilisation de la GATPA pour chaque parturiente. Les utérotoniques utilisés sont l'ocytocine en intramusculaire ou le misoprostol par voie rectale. Trente-quatre pourcent de la mortalité maternelle est imputée à l'’hémorragie du post-partum. (24)

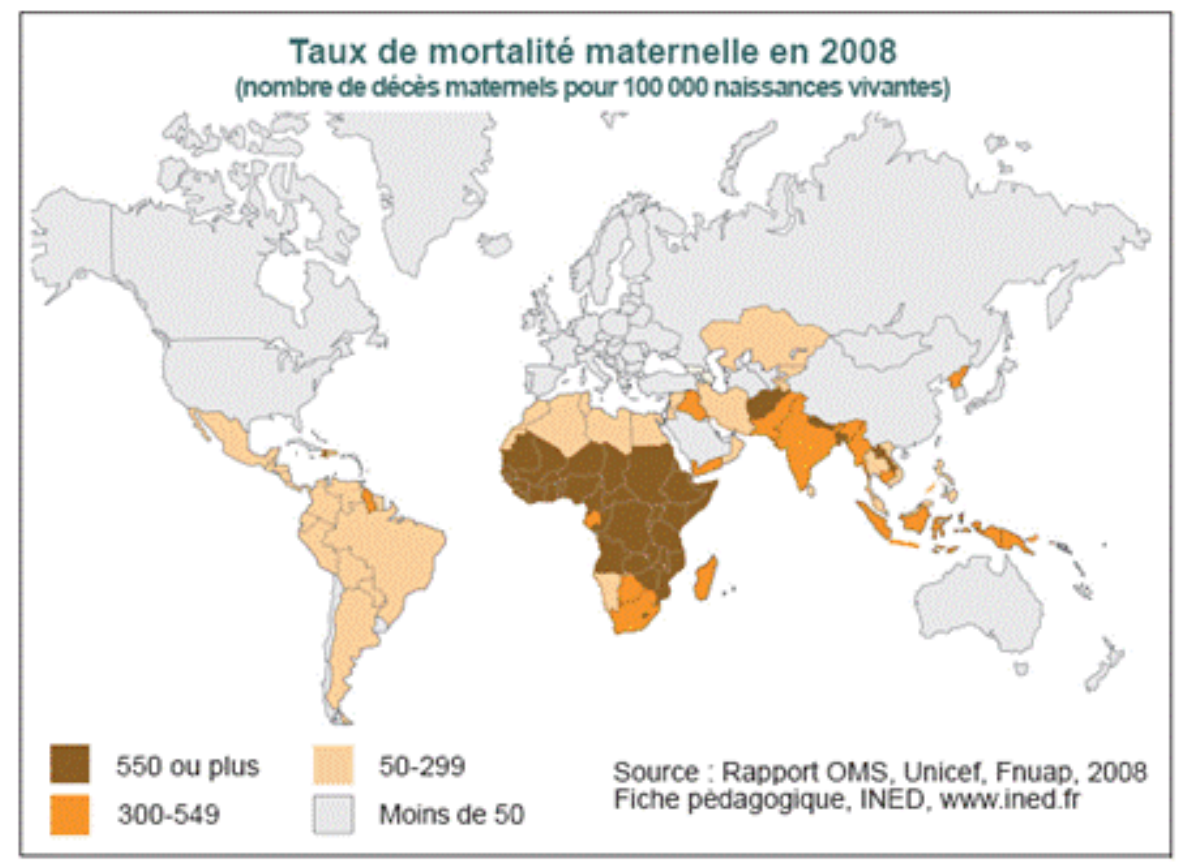

Figure 1 : Mortalité maternelle dans le monde en 2008

\section{2. $\mathrm{Au} \mathrm{Togo}$}

\section{a. Politique de santé togolaise}

Selon le rapport de 2002 sur les comptes nationaux de santé, les dépenses annuelles de santé s'élèvent à 121 milliards de francs CFA soit environ 185 millions d'euros. 55\% de ces dépenses sont concentrées dans la commune de Lomé (la capitale).

Le secteur privé représente plus de $80 \%$ des dépenses de santé. Ces dépenses sont à $83 \%$ financées par les ménages et $1.6 \%$ par les fonds de sécurité sociale. La sécurité sociale togolaise appelé Institut national d'assurance maladie (INAM) n'a été mise en place que depuis quelques années et seuls les fonctionnaires peuvent en être bénéficiaires. Or, les fonctionnaires ne représentent que 
$0,7 \%$ de la population. Donc très peu de patients sont concernés par une prise en charge de leurs dépenses de santé (25) (ANNEXE III).

Il existe aussi des assurances privées prenant en charge certaines dépenses de santé. Mais bien entendu, seules les personnes ayant un bon niveau économique peuvent y souscrire.

\section{b. Epidémiologie de l'HPPI}

En 1992, une étude rapporte une fréquence d'HPPI au Togo de 0,89\% (26), ce qui parait très inférieur aux 5\% d'HPPI généralement retrouvés dans la littérature internationale. En 2002, la fréquence des hémorragies du post-partum était évaluée à 6,89\%. En l'absence d'outil de recueil épidémiologique, la variabilité des taux d'HPP d'une publication à l'autre souligne la difficulté à obtenir des données fiables à l'échelle d'un service et à plus forte raison à l'échelle d'un pays. L'atonie utérine était responsable de 54\% de ces HPPI; Dans $78 \%$ des cas, la prise en charge thérapeutique de ces hémorragies a été limitée aux gestes obstétricaux classiques (révision utérine, massage utérin et ocytocique, traction sur le col et clampage des artères utérines). Quatorze pourcent des patientes ont été transfusées et $4 \%$ ont subi une hystérectomie d'hémostase.

En 2006, le taux rapporté est de 1,36\%. L'étude réalisée en 2007 sur une période de 20 ans (19872007) au CHU de Lomé par l'équipe du professeur Apkadza ne montre pas de grande diminution du taux de décès maternel suite à une HPP. La population la plus touchée demeure les jeunes femmes âgées de 25 à 29 ans avec un bas niveau d'instruction et provenant d'une autre structure sanitaire. (27)

Les conclusions de cette étude sont la nécessité d'une «subvention des soins obstétricaux d'urgence» et «étendre la couverture sanitaire ». La prise en charge de l'HPPI au sein de la maternité du CHU-SO suit les Recommandations pour la pratique clinique (RPC) des soins obstétricaux et néonataux d'urgence en Afrique. Ces recommandations sont publiées par l'OMS Afrique en partenariat avec la Société Africaine des Gynécologues-obstétriciens (SAGO) (ANNEXE IV).

\section{PROBLEMATIQUE :}

Quels sont les moyens de prévention et de prise en charge de l'HPPI au CHU-SO de Lomé ? Ces moyens sont-ils conformes aux recommandations de l'Organisation mondiale de la santé (OMS) et de la Fédération internationale des gynécologues-obstétriciens (FIGO) ? 


\section{Deuxième partie: Méthodologie}

\section{Objectifs et hypothèses}

\section{Objectifs de l'étude}

L'objectif de cette étude était d'évaluer les pratiques de prévention et de prise en charge de l'hémorragie du post-partum au Togo.

Elle s'inscrit dans une démarche d'amélioration de la prise en charge afin de réduire la morbidité et la mortalité maternelle au Togo.

L'objectif principal était donc de décrire les pratiques quotidiennes des professionnels de l'obstétrique du C.H.U Sylvanus Olympio de Lomé dans la prévention et la prise en charge de l'HPPI et de voir si ces pratiques sont conformes aux recommandations internationales de l'Organisation mondiale de la santé (OMS) et de la Fédération internationale des gynécologues et obstétriciens (FIGO).

Les objectifs secondaires étaient d'évaluer la formation du personnel, et enfin d'identifier des différents modes de prise en charge de l'HPPI s'il existe un différence de pratiques selon les professionnels.

\section{Hypothèses de recherche}

La sévérité de l'hémorragie du post-partum immédiat (HPPI) au Togo peut être la conséquence de plusieurs facteurs :

- Le système de soins périnataux :

- L'accès aux soins des femmes enceintes.

- La qualité des soins dispensés par les professionnels de santé. 
- L'organisation des soins au CHU-SO :

- Le personnel (formation suivie).

- La gestion du travail

- Une définition de l'HPPI différente selon les professionnels.

- Les protocoles de prévention et de prise en charge de l'HPPI non conformes aux recommandations internationales.

- Un accès limité aux produits sanguins labiles.

- Un manque d'équipement et de matériels adaptés.

\section{Méthodologie de l'enquête}

\section{Type d'enquête, lieu et durée}

Nous avons mené une étude descriptive au sein de la maternité du C.H.U Sylvanus Olympio. Il s'agit de l'hôpital public de référence sur l'ensemble du territoire national. Cette étude a porté sur la période du $1^{\mathrm{er}}$ juillet au 19 juillet 2013 soit une durée de trois semaines.

Cette étude reposait sur un recueil de donnés à partir de dossiers obstétricaux, du cahier d'accouchement du bloc opératoire et de l'observation. A ces données s'ajoute un entretien semidirectif avec six professionnels : 3 sages-femmes et 3 obstétriciens.

\section{Population étudiée}

A été inclus dans cette étude, tout accouchement par voie basse ou césarienne à un terme $\geq 24$ semaines d'aménorrhées. Y sont aussi incluses les patientes ayant accouché en dehors du centre d'étude mais y ayant été transférées pour HPPI. 


\section{Variables analysées dans l'étude descriptive de la prévention et de la prise en charge de l'HPPI}

Pendant la période d'étude, les données démographiques, les modalités du travail obstétrical, de l'accouchement, de la délivrance et de la surveillance du post-partum immédiat des patientes prises en charge à la maternité ont été recueillies à partir des dossiers «papier» des patientes ou de l'observation directe en salle de naissance.

Les paramètres analysés sont décrits ci-après.

\section{a. Caractéristiques générales et obstétricale}

L'âge, la gestité et la parité des patientes ont été relevées lorsqu'elles sont connues.

Une patiente est dite grande multipare si elle a accouché d'au moins 5 enfants.

Les antécédents de césarienne et de myomectomie ont aussi été pris en compte.

\section{b. Grossesse actuelle}

Le nombre de foetus a été noté. Une grossesse multiple est définie par la présence d'au moins deux fœtus.

Le suivi de la grossesse a été pris en compte. La réalisation d'au moins 4 consultations prénatales définit un suivi régulier de la grossesse.

Les pathologies gravidiques telles que l'hypertension artérielle, la pré-éclampsie, une anomalie d'insertion placentaire, ainsi que l'existence de troubles de l'hémostase ont été recherchées.

\section{c. Déroulement du travail et de l'accouchement}

- Le terme et la voie d'accouchement

- Les moyens de mise en travail: spontané, déclenchement (par ocytocine, ou misoprostol)

- Utilisation de Syntocinon® pendant le travail

- Durée du travail (travail long $\geq 10 \mathrm{~h}$, travail rapide $<5 \mathrm{~h}$ )

- Type de délivrance

- GATPA (Gestion active de la troisième phase de l'accouchement) 


\section{d. Le post-partum}

- L'utérotonique administré de manière préventive

- La durée et la fréquence de la surveillance

\section{e. La prise en charge de l'hémorragie du Post-partum Immédiat}

- L'étiologie de l'hémorragie lorsqu'elle est connue

- La prise en charge mise en place : traitement initial, utérotonique thérapeutique utilisé, transfusion, prise en charge chirurgicale.

- Pour les patientes transférées pour HPPI le délai de transfert a été calculé lorsque l'heure de l'accouchement est connue.

\section{Variables analysées par l'étude qualitative}

La part qualitative de cet écrit repose sur des entretiens individuels avec six professionnels de la périnatalité, travaillant au sein du centre d'étude : 3 sages-femmes et 3 obstétriciens. Ces entretiens portent sur les connaissances et les protocoles de prévention et de prise en charge de l'HPPI (Hémorragie du post-partum immédiat) ainsi que sur le ressenti personnel par rapport au nombre élevé d'HPPI en Afrique.

Les critères évalués étaient :

- Formation suivie

- Définition et causes d'HPPI

- Prise en charge de l'HPPI

- Gestion antérieure d'HPPI et devenir des patientes

- Ressenti des professionnels face aux taux élevés d'HPPI en Afrique ainsi que les moyens de prévention à mettre en place pour réduire ce nombre. 


\section{Troisième partie : Résultats}

\section{Description du système de soins au Togo et du circuit du patient en maternité}

Le système de soins au Togo, comme dans de nombreux pays en développement, est basé sur la participation financière des patients ainsi que sur la participation des familles aux soins du malade, en l'absence de financement public de la santé comme en France.

Le fonctionnement de l'hôpital, les salaires du personnel médical et paramédical sont financés par l'état, mais le patient paye des frais de séjour forfaitaires à hauteur de 7500FCFA $(11,50 €)$.

Concernant le circuit du patient en maternité, la parturiente est accueillie par la sage-femme des urgences. Comme à chaque consultation, la patiente doit présenter son carnet de suivi de grossesse, dans lequel sont contenus ses antécédents, et l'ensemble des éléments de suivi de la grossesse (compte rendu d'échographie si faite, résultats de bilans sanguins, consultations antérieures).

La patiente est suivie au CHU Sylvanus Olympio, ou provenant d'un autre centre médical (clinique privée, maisons d'accouchements, centre médico-social) en cas de pathologie. Dans ce dernier cas, la patiente est dite référée.

Selon le motif de consultation de la patiente et surtout selon l'urgence qui en découle, la patiente est pesée et mesurée. Mais dans tous les cas, un examen général et obstétrical est réalisé. Il comprend la mesure de la tension artérielle, l'examen des conjonctives et la réalisation d'une bandelette urinaire. Les patientes ramènent à chaque consultation un petit flacon en verre ou en plastique dans lequel elles urinent à l'arrivée à la maternité. Dans le cas échéant, elles sont tenues d'en acheter un aux professionnels de santé ; ainsi, pour chaque patiente, une bandelette urinaire est obligatoirement réalisée. Dans le cas où la patiente présente un état général altéré (c'est souvent le cas des patientes transférées pour éclampsie), la bandelette urinaire est réalisée en salle de naissances par un sondage urinaire. 
La prise de la température ne peut se faire que si la patiente dispose d'un thermomètre personnel. L'examen obstétrical comprend la mesure de la hauteur utérine à l'aide d'un mètre-ruban, l'audition de bruits du cœur du fœetus à l'aide d'un stéthoscope de pinard et enfin la réalisation d'un toucher vaginal.

En fonction des résultats de cet examen, la patiente est renvoyée chez elle avec des conseils d'usages ou est hospitalisée.

Lorsque la patiente est hospitalisée et que son état nécessite une prise en charge immédiate (accouchement imminent, éclampsie, hémorragie du post-partum...), elle est directement dirigée vers la salle d'accouchements où elle est rapidement prise en charge par les sages-femmes et les médecins de garde (en cas de pathologies graves). Dans le cas où l'état de la patiente ne constitue pas une urgence vitale ni pour elle, ni pour l'enfant à naître, (Début de travail, pré-éclampsie modérée, rupture prématurée des membranes à terme...) elle est dirigée vers la salle de travail où elle sera surveillée par la sage-femme de la salle de travail et vue plus tard par un médecin si nécessaire.

Ainsi, la plupart des patientes, qui se présentent aux urgences gynécologiques pour des contractions utérines et qui sont en début de travail, passent par la salle de travail et n'ont accès à la salle d'accouchements que lorsque le travail obstétrical arrive à sa fin (en moyenne à une dilatation cervicale de $7-8 \mathrm{~cm})$.

Il existe une «salle d'attente des césariennes », pour les patientes admises pour une césarienne programmée.

Enfin, dans le cas où la patiente n'est pas à terme, elle est dirigée vers le service des grossesses à haut risque.

Une ordonnance est remise à la famille de la patiente en fonction de la voie d'accouchement décidée à l'arrivée en salle.

Dans le cas d'un accouchement voie basse, un kit voie basse est prescrit. Il contient : 2paires de gants stériles (taille 8 ou 7.5), une seringue, une aiguille, un cathéter, une tubulure, un flacon de $500 \mathrm{cc}$ de sérum physiologique, 30 à 40 UI d'ocytocine.

Le kit de césarienne contient le nécessaire pour la rachianesthésie, de l'ocytocine, des solutés, des compresses, des gants stériles, 1 flacon de Bétadine, un pansement, des fils.

En fonction de l'évolution de l'état de la patiente, les sages-femmes font souvent appel aux accompagnants de la patiente, pour aller chercher des médicaments dans les pharmacies alentours dans les plus brefs délais. Dans les cas où, les familles ne peuvent pas rapidement honorer les prescriptions, et qu'il s'agit d'une urgence, il arrive souvent que les sages-femmes, disposant ellesmêmes d'un stock de médicaments comme par exemple le Syntocinon® et le Cytotec®, l'administrent à la patiente en attendant le retour du membre de la famille. 
Pour tout accouchement par voie basse, la Gestion active de la troisième phase de l'accouchement (GATPA) est réalisée.

La patiente est perfusée juste après l'accouchement afin de lui administrer l'ocytocine durant la surveillance du post-partum immédiat.

Après la toilette périnéale, la patiente est surveillée pendant un minimum de $2 \mathrm{~h}$ soit dans la salle d'accouchement mais le plus souvent, dans la salle des accouchées mitoyenne, avant d'aller dans le service des suites de couches. Les accouchées sont hospitalisées en moyenne 3 jours après l'accouchement.

Dans le cas d'une césarienne, la patiente est admise en réanimation où elle est surveillée pendant en moyenne $24 \mathrm{~h}$. L'autorisation de sortie de la réanimation vers les suites de couches est donnée par le médecin de garde qui y fait sa visite chaque jour. Pendant la période de surveillance, le nouveauné est gardé par la famille de la patiente (une femme) dans la chambre des «bébés césarienne». Dans le cas de patientes qui allaitent, un membre de la famille lui amène le bébé, le temps de la tétée. Les patientes césarisées sont gardées en moyenne 5 jours. 


\section{Résultats de l'analyse descriptive}

\section{Prévention de l'hémorragie du post-partum immédiat (HPPI)}

Durant la période d'étude du $1^{\text {er }}$ juillet au 19juillet 2013, nous avons pu regrouper 300 dossiers de patientes admises à la maternité du CHU Sylvanus Olympio pour un accouchement ou pour une pathologie du post-partum immédiat. Sur ces 300 patientes, 8 n'ont été transférées au centre d'étude qu'après l'accouchement, pour une HPPI.

\section{a. Caractéristiques des patientes admises en travail}

Le tableau ci-après regroupe les caractéristiques des patientes ayant accouché au CHU-SO ; soit 292 patientes.

\begin{tabular}{lcc} 
Critères & $\begin{array}{c}\text { Moyenne ou } \\
\text { nombre(n) }\end{array}$ & $\begin{array}{c}\text { Limites ou } \\
\%\end{array}$ \\
\hline Age (ans) & 27 & $17-43$ \\
& & \\
Parité (n ; \%) & 119 & 39.7 \\
-primiparité & 145 & 48.3 \\
-multiparité & 36 & 12 \\
-grande & & \\
multiparité ( $\geq 5)$ & 163 & 54.3 \\
Suivi au CHU & 132 & 44 \\
-oui & 5 & 1.7 \\
-non & 39 & $33-44$ \\
-pas de données & & \\
Terme (SA) & & \\
\hline
\end{tabular}

Tableau 1: caractéristiques des patientes admises en travail

Quarante-quatre pourcent des patientes n'ont pas été suivies au centre d'étude durant leur grossesse. Elles proviennent de centres médico-sociaux (CMS) ou de cliniques privées, et ont été transférées pendant le travail pour une meilleure prise en charge. 


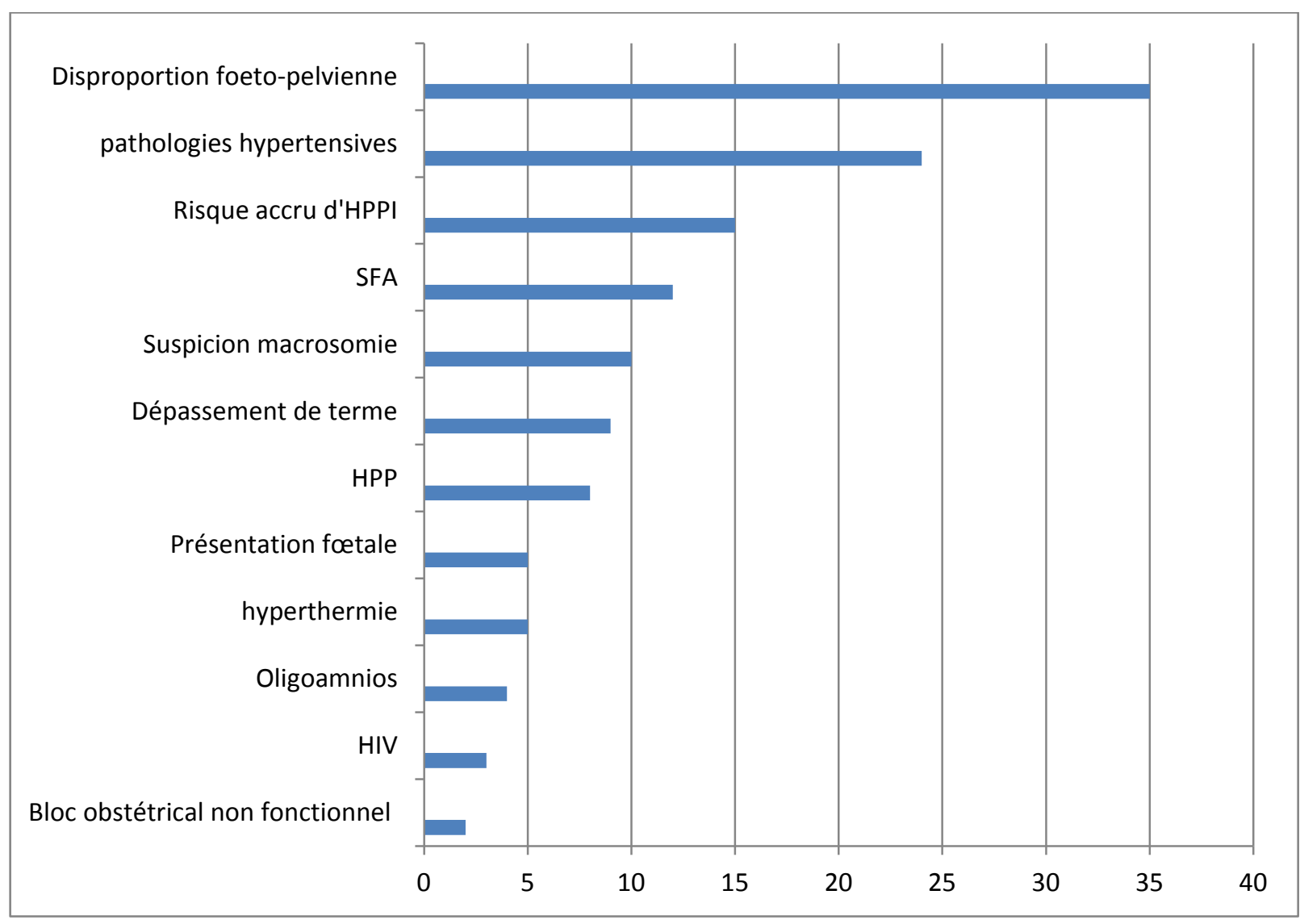

Graphique 1: Motifs d'admission des patientes transférées pendant le travail

Le motif principal de transfert des patientes est une suspicion de disproportion foeto-pelvienne se traduisant par une stagnation de la dilatation cervicale. On retrouve aussi les pathologies hypertensives (HTA sévère isolée, pré-éclampsie, éclampsie) qui constituent le deuxième motif d'admission de patientes suivies à l'extérieur.

Les motifs de transfert des patientes considérées comme ayant un risque accru d'HPPI sont : une suspicion de décollement placentaire, hypercinésie utérine, métrorragies, anomalie d'insertion placentaire, déhiscence de l'ancienne cicatrice, utérus cicatriciel et enfin un âge maternel élevé (38ans) associé à un utérus cicatriciel. Ces patientes représentent 11,36\% des patientes transférées.

Ces motifs de transfert constituent un facteur de risque d'HPPI, auxquels s'ajoutent d'autres critères, explicités dans le tableau ci-dessous. 
Critères $\quad$ Nombre $(n=292) \quad$ Pourcentage $(97.3 \%)$

\begin{tabular}{lcc}
\hline Utérus cicatriciel $(\mathbf{n}$;\%) & 43 & 14.5 \\
-uni cicatriciel & 21 & 7.1 \\
-bi cicatriciel & $1_{1}$ & 0.3 \\
Troubles de l'hémostase & 9 & \\
Suivi de la grossesse & 89 & 3 \\
-aucun suivi & 188 & 29.7 \\
-peu de suivi & 14 & 62.6 \\
-suivi régulier (>=4 CPN) & 10 & 4.7 \\
-pas de données & 42 & 3.4 \\
Placenta prævia & & 1.3 \\
Grossesse multiple & 3 & \\
Pathologie de la grossesse & 41 & 1 \\
-drépanocytose & 1 & 13.7 \\
-HTA isolée & 7 & 0.3 \\
-myome prævia & 3 & 2.3 \\
-pré-éclampsie & & 1 \\
-paludisme & & \\
\hline
\end{tabular}

1: Trouble de l'hémostase : thrombopénie à 68000plaquettes

2: Quatre grossesses multiples dont une grossesse triple

Tableau 2 : Etude des facteurs de risque d'HPPI préexistant au travail

Plus de $21 \%$ des patientes présentaient un utérus cicatriciel à l'admission.

La majorité des patientes admises ont été suivi durant leur grossesse. Ainsi, près de $62.7 \%$ des parturientes ont eu au minimum 4 consultations prénatales conformément aux recommandations de l'Organisation mondiale de la santé (OMS). Trois pourcent n'ont eu aucun suivi et se sont présentées tardivement à la maternité, le jour de l'accouchement.

Nous n'avons pu relever qu'un seul cas d'anomalie de l'hémostase du fait de la quasi absence du suivi biologique ou du moins de la traçabilité de ce suivi dans les dossiers d'accouchement.

Enfin, 18.3\% des patientes ont présenté une pathologie pendant la grossesse. Dans la majorité des cas, il s'agissait de pathologies hypertensives.

Donc, les patientes admises au CHU, sont majoritairement des jeunes femmes, multipares ayant eu un suivi régulier de leur grossesse et à priori sans pathologies. 


\section{b. La gestion du travail et de l'accouchement}

Comme vu, dans la première partie, la gestion du travail obstétrical peut constituer un facteur de risque d'HPPI.

L'analyse de la gestion du travail se fera sur un total de 239 patientes ; car sur les 300 patientes, 292 ont accouché au CHU, parmi lesquelles 53, ont eu une césarienne avant le début du travail.

\begin{tabular}{lll} 
Caractéristiques du travail & $\mathrm{n}(=239)$ & $\%$ \\
\hline Mode d'entrée en travail & & \\
-spontané & 208 & 87 \\
-déclenchement & 22 & 9.2 \\
- absence de données & 9 & 3.8 \\
Direction du travail au Syntocinon $^{\circledR}$ & 28 & 11.7 \\
Durée du travail & & \\
-rapide & 127 & 53.1 \\
-normal & 71 & 29.7 \\
-long & 32 & 13.4 \\
-absence de données & 9 & 3.8 \\
\hline
\end{tabular}

\section{Tableau 3 : Modalités du déroulement du travail}

La dilution du Syntocinon ${ }^{\circledR}$ se fait comme en France c'est-à-dire 5 UI dans 500cc de solution glucosée à 5\%. Le débit est fixé à 8 gouttes/minutes (ce qui correspond à $0.4 \mathrm{ml} / \mathrm{h}$ ). Seules $11,7 \%$ des patientes ont eu un travail dirigé au Syntocinon®.

En ce qui concerne la durée de travail, les patientes ont majoritairement (à 53.3\%) eu un travail rapide c'est-à-dire d'une durée de moins de 5 h. Mais du fait de la rareté de l'utilisation du partogramme par les sages-femmes (par manque de temps), la dynamique du travail notamment pour les patientes ayant eu un travail long n'a pu être étudié.

\begin{tabular}{lcc} 
CRITERES & $\mathrm{n}(=292)$ & $\%$ \\
\hline Voie d'accouchement & 180 & 61.6 \\
-Voie basse & 53 & 18.2 \\
-Césarienne avant travail & 59 & 20.2 \\
-Césarienne en urgence & & \\
Total & 292 & 100 \\
\hline
\end{tabular}

Tableau 4: Modalités d'accouchement 
L'étude des modalités d'accouchement prend en compte les patientes ayant eu une césarienne avant l'entrée en travail.

Les indications de césarienne avant travail étaient le plus souvent un utérus bi-cicatriciel, une prééclampsie sévère avec des conditions locales défavorables, une présentation fœtale inadaptée ou présentant un risque important à la voie basse (la présentation transverse et la présentation du siège chez une primipare), une grossesse multiple ou une sérologie VIH positive.

Les motifs de césarienne pendant le travail, étaient le plus souvent pour anomalies du rythme cardiaque fotal mais aussi dans certains cas pour des pathologies maternelles le plus souvent caractérisée par des pathologies hypertensives graves comme l'éclampsie.

\section{c. La délivrance : troisième phase de l'accouchement}

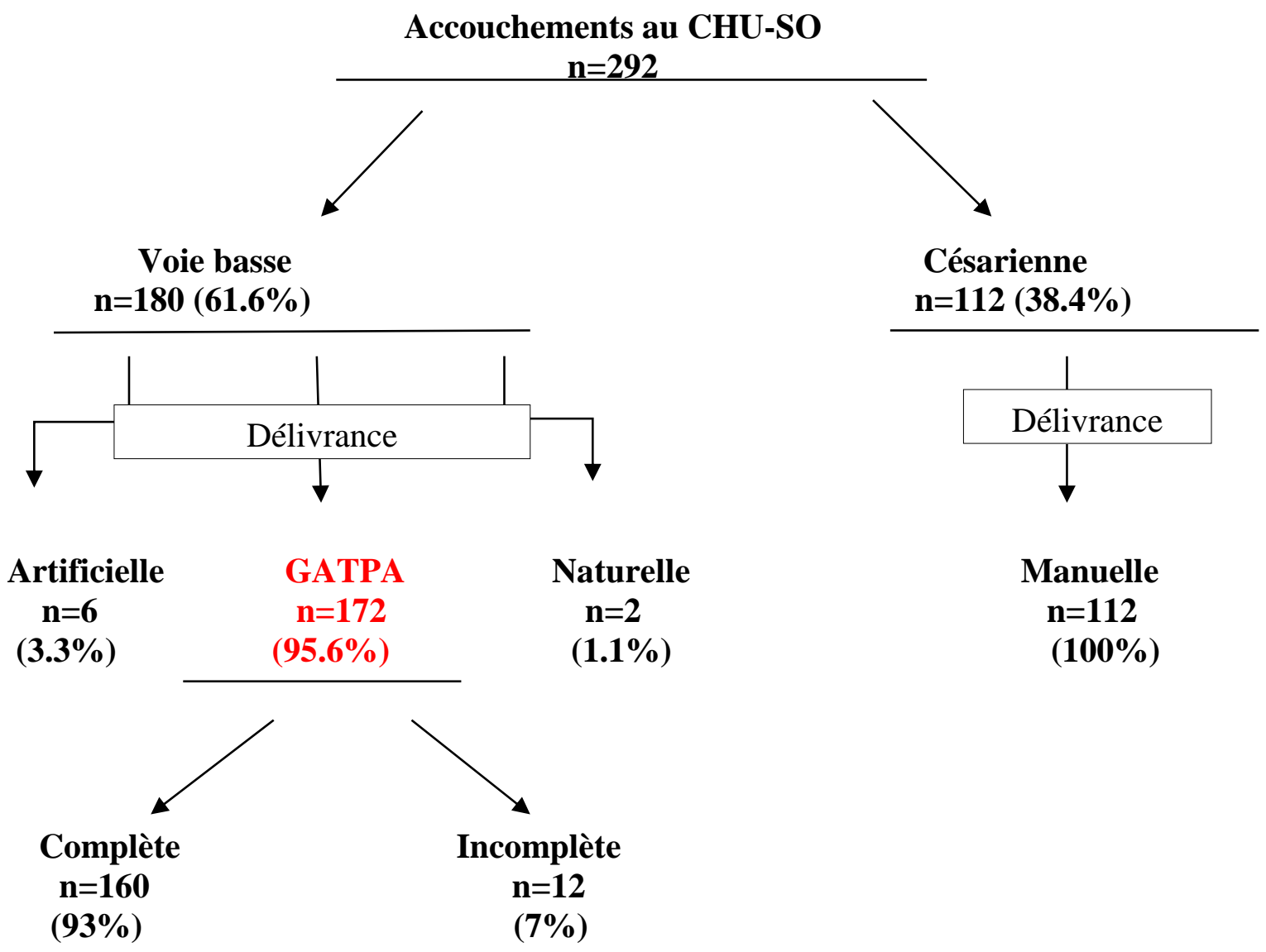

Figure 2 : Modalités de la délivrance 
L'analyse des modalités de la délivrance a été faite sur les patientes ayant accouché au CHU-SO et plus particulièrement sur les accouchements par voie basse afin de mettre en évidence la fréquence de la pratique de la GATPA

Sur les 180 accouchements par voie basse, la gestion active de la troisième phase de l'accouchement (GATPA) a été pratiquée à 95,6\%.

Lors de tous les accouchements auxquels j'ai pu assister, la GATPA a été pratiquée par la sagefemme ou l'obstétricien ayant réalisé l'accouchement.

Les sages-femmes de la salle de naissance disposent d'un stock personnel d'ocytocine de synthèse. Ainsi, lorsque la patiente accouche avant qu'un membre de la famille ne rapporte le kit d'accouchement, elle bénéficie tout de même de la GATPA.

Dans le cadre de la GATPA, la délivrance est dite « assistée » (analogue de la délivrance dirigée)

Lorsque la délivrance a été incomplète, difficile et /ou retardée une révision utérine est rapidement réalisée par la sage-femme.

Une délivrance artificielle et une révision utérine est très souvent pratiquée systématiquement en cas de mort fœtale in utéro en prévention d'une HPPI. Ainsi, sur sept décès fœtaux in utéro, six délivrances artificielles suivies d'une révision utérine ont été pratiquées, soit 85.7\%.

\section{d. Surveillance du post-partum immédiat}

\begin{tabular}{lcc}
\multicolumn{1}{c}{ CRITERES } & $\mathrm{n}$ & $\%$ \\
\hline Utérotonique dans le post-partum & $=292$ & \\
-Ocytocine seul & 231 & 79.1 \\
-Ocytocine+misoprostol & 57 & 19.5 \\
-Ocytocine+Méthergin ${ }^{1}$ & 4 & 1.4 \\
Surveillance du post-partum & & \\
-<2 heures & 1 & 0.3 \\
-22 heures & 144 & 49.3 \\
-Absence de données & 147 & 50.4 \\
Fréquence de la surveillance & & \\
-Au moins 1 fois & 107 & 36.6 \\
-Au moins 3 fois & 66 & 22.6 \\
-Au moins 4 fois & 25 & 8.6 \\
-Absence de données & 94 & 32.2 \\
Examen médical & & \\
-Oui & 170 & 58.2 \\
-Absence de données & 122 & 41.8 \\
\hline
\end{tabular}

Tableau 5 : Le post-partum immédiat 
L'étude de la surveillance du post-partum immédiat a été réalisée sur le nombre total d'accouchées y compris les césariennes, soit sur 292 dossiers.

L'utérotonique le plus utilisé est l'ocytocine de synthèse. Il a été utilisé chez toutes les patientes quelle que soit la voie d'accouchement. Dans certaines situations de risque accru de saignements, il est souvent associé au misoprostol en prévention d'une HPPI. Cinq comprimés de misoprostol (soit $1000 \mu \mathrm{g}$ ) sont administrés à la patiente par voie intra-rectale.

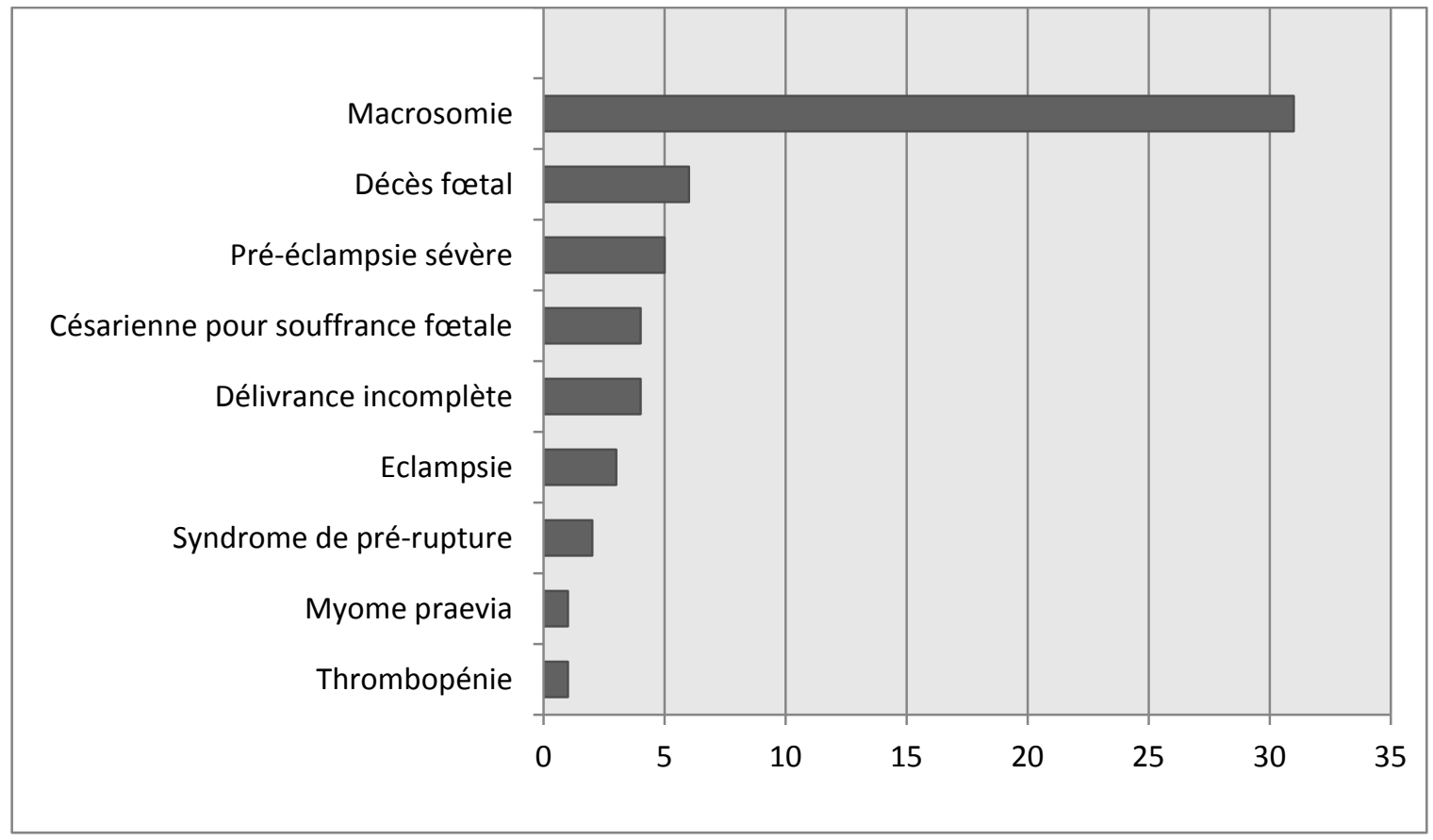

\section{Graphique 2 : Patientes ayant reçu du misoprostol en intra-rectal}

Le misoprostol a été administré dans $54.4 \%$ aux patientes ayant accouché d'un enfant macrosome. La patiente ayant présenté une thrombopénie ainsi que les patientes ayant accouché d'un enfant mort in utéro ont elles aussi bénéficié d'un traitement préventif associant une perfusion d'ocytocine et du misoprostol par voie rectale.

Les patientes césarisées étant gardées en salle de réanimation durant en moyenne les 24 premières heures post-opératoire, elles bénéficient d'une surveillance plus accrue. Ainsi, les patientes ayant eu au moins 3 surveillances annotées de leurs saignements de la tonicité utérine et/ou une mesure de la tension artérielle sont principalement des césariennes. 


\section{Prise en charge de l'hémorragie du post-partum immédiat}

En cas d'HPPI, le médecin de garde est appelé par la sage-femme après que cette dernière ait réalisé une révision utérine et un massage utérin dans le but d'arrêter les saignements excessifs. A l'arrivée du médecin, ce dernier réalise une nouvelle fois une révision utérine et en fonction de la quantité des pertes sanguines et de la cause suspectée d'hémorragie, il met en place une conduite à tenir.

J'ai pu observer personnellement un cas de prise en charge d'une HPPI due à une déchirure cervicale. Immédiatement, la sage-femme pratiqua une révision utérine, un sondage vésical évacuateur et un massage utérin. Devant la quantité des pertes sanguines, le médecin fut appelé 10 minutes après la délivrance. De même une révision utérine fut faite par le chef de garde qui perçut au toucher la déchirure cervicale. La décision fut donc prise de faire une révision du col sous valves au bloc opératoire. Or toutes les salles de bloc opératoire étaient occupées; et la révision n'a pu être faite qu' $1 \mathrm{~h} 20$ après l'accouchement sous anesthésie générale. Les pertes sanguines totales ont été estimées à $1200 \mathrm{cc}$. La patiente bénéficia de 20UI de Syntocinon® dans le post-partum immédiat associé à $1000 \mu \mathrm{g}$ de Cytotec ${ }^{\circledR}$ par voie rectale.

Les autres cas ci-après sont décrits à partir des données des dossiers obstétricaux. Durant la période d'étude, nous avons relevé 14 cas d'HPPI soit un pourcentage de $4.7 \%$.

L'âge moyen de ces patientes est de 28ans (la plus jeune ayant 23 ans et la plus âgée 40 ans).

Du fait de la sous-estimation des pertes sanguines, nous ne pourrons pas prendre comme définition de référence une perte sanguine $\geq 500 \mathrm{ml}$ lors d'un accouchement par voie basse et une perte $\geq 1000 \mathrm{ml}$ lors d'une césarienne. Ainsi donc, les 14 cas d'hémorragie du post-partum, sont les patientes ayant été transfusées suite à l'accouchement et/ou ayant été transférées pour hémorragie du post-partum immédiat.

Parmi ces 14 patientes, six ont accouché au centre d'étude et huit y ont été transférées pour HPPI. L'analyse de la prise en charge des HPPI se fera en deux temps : la prise en charge des patientes ayant accouché au centre d'étude puis la prise en charge des patientes transférées.

\section{a. Caractéristiques et prise en charge des patientes ayant accouchées au CHU-SO}

Les patientes ayant présenté une HPPI après un accouchement sont majoritairement des patientes jeunes et ayant eu un suivi régulier pendant leur grossesse. Mais seul le tiers de ces patientes a été suivi au centre d'étude pendant la grossesse. 


\begin{tabular}{llc}
\hline Age (ans) & & \\
$-<35$ ans & 4 & $66.7(28.6)$ \\
$-\geq 35$ ans & 2 & $33.3(14.3)$ \\
Parité & & \\
-Primiparité & 3 & $50(21.4)$ \\
-Multiparité & 2 & $33.3(14.3)$ \\
-grande multiparité & 1 & $16.7(7.1)$
\end{tabular}

\section{Suivi au CHU-SO}

-Oui

-Non

Motifs d'admissions

-césarienne prévue

-Contractions utérines

$33.3(14.3)$

-Placenta Prævia

1

$33.3(14.3)$

hémorragique

-Suspicion macrosomie

\section{Etat des conjonctives}

-Colorées

4

-Absence de donnés

2

\section{Suivi de grossesse}

-peu de suivi

-Suivi régulier

\section{Mode d'entrée en travail}

-Spontané

-pas de travail

66.7 (28.6)

\section{Voie d'accouchement}

-Voie basse

-Césarienne en urgence

-Césarienne avant travail

2

$33.3(14.3)$

$33.3(14.3)$

\section{Délivrance}

-Assistée complète

-Manuelle

Tableau 6: Caractéristiques des patientes ayant présenté une HPPI après un accouchement au CHU-SO 
A l'admission, quatre soit $66,7 \%$ présentaient un facteur de risque important d'HPPI : un utérus polymyomateux avec un myome prævia, une anomalie de l'insertion placentaire et une suspicion de macrosomie qui fut confirmée à la naissance $(4800 \mathrm{~g})$. Aucune patiente n'a reçu de l'ocytocine pendant le travail.

\begin{tabular}{|c|c|c|c|c|c|c|c|}
\hline Patientes & $\begin{array}{l}\text { Cause } \\
\underline{\text { HPPI }}\end{array}$ & $\begin{array}{l}\text { Délai du } \\
\text { diagnostic }\end{array}$ & $\begin{array}{l}\text { Traitement } \\
\text { initial }\end{array}$ & Utérotonique & $\begin{array}{l}\text { Délai } \\
\text { transfusion }\end{array}$ & $\begin{array}{l}\text { Pertes } \\
\text { estimées } \\
\end{array}$ & $\begin{array}{l}\frac{\text { Traitement }}{\text { chirurgical }} \\
\angle \text { Délai }\end{array}$ \\
\hline $\begin{array}{l}40 \text { ans, } 8^{\text {ème }} \\
\text { Placenta } \\
\text { prævia }\end{array}$ & $\begin{array}{l}\text { Non } \\
\text { connue }\end{array}$ & Immédiat & Non connu & $\begin{array}{l}5 \text { cps* de } \\
\text { Cytotec }{ }^{*} \\
1 \text { mg de } \\
\text { Méthergin } \AA\end{array}$ & $\begin{array}{l}\text { Non } \\
\text { transfusée }\end{array}$ & $150 c c$ & $\begin{array}{l}\text { Ligature } \\
\text { vasculaire } \\
\text { (ronds et } \\
\text { utéro- } \\
\text { ovariens) }\end{array}$ \\
\hline $\begin{array}{l}40 \text { ans, } \\
\text { myome } \\
\text { prævia }\end{array}$ & $\begin{array}{l}\text { Non } \\
\text { connue }\end{array}$ & $2 \mathrm{~h} 00$ & $\begin{array}{l}\text {-Révision } \\
\text { utérine(RU), } \\
\text { massage } \\
\text { utérin }\end{array}$ & Non connu & $\begin{array}{l}\text { 7h00 } \\
\text { 1PFC*+ } \\
\text { 2CGR* }\end{array}$ & $1300 \mathrm{cc}$ & $\begin{array}{l}\text { Ligature } \\
\text { vasculaire et } \\
\text { hystérectomie } \\
\text { Délai : } 45 \mathrm{~min}\end{array}$ \\
\hline $\begin{array}{l}\text { Placenta } \\
\text { prævia }\end{array}$ & $\begin{array}{l}\text { Non } \\
\text { connue }\end{array}$ & Immédiat & Non connu & $\begin{array}{l}\text { cps } \\
\text { Cytotec }{ }^{\circ} \\
1 \mathrm{mg} \text { de } \\
\text { Méthergin } \AA\end{array}$ & $\begin{array}{l}\text { 3h15 } \\
1 \mathrm{PFC}^{*}+ \\
1 \mathrm{CGR}^{*}\end{array}$ & $150 c c$ & Non \\
\hline $\begin{array}{l}\text { Grossesse } \\
\text { peu suivie } \\
\text { au SO }\end{array}$ & $\begin{array}{l}\text { Déchirure } \\
\text { cervicale }\end{array}$ & Immédiat & $\begin{array}{l}\mathrm{RU}, \\
\text { massage } \\
\text { utérin, } \\
\text { sondage, }\end{array}$ & $\begin{array}{l}5 \mathrm{cps} \\
\text { Cytotec } \AA\end{array}$ & $\begin{array}{l}\text { Non } \\
\text { transfusée }\end{array}$ & $1200 c c$ & $\begin{array}{l}\text { Suture du col } \\
\text { sous valves } \\
\text { Délai : 1h20 }\end{array}$ \\
\hline Macrosomie & $\begin{array}{l}\text { Atonie } \\
\text { utérine }\end{array}$ & $1 \mathrm{~h} 10$ & Idem & Non connu & $\begin{array}{l}\text { 1CGR } \\
\text { Délai non } \\
\text { connu }\end{array}$ & $>700 \mathrm{cc}$ & Non \\
\hline $\begin{array}{l}\text { Pas de } \\
\text { facteurs } \\
\text { particuliers }\end{array}$ & $\begin{array}{l}\text { Atonie } \\
\text { utérine }\end{array}$ & Immédiat & $\begin{array}{l}\mathrm{RU}, \\
\text { massage, } \\
\text { sondage }\end{array}$ & $\begin{array}{l}5 \text { cps de } \\
\text { Cytotec } \AA\end{array}$ & $\begin{array}{l}\text { 6h00 } \\
1 \text { CGR }\end{array}$ & $\begin{array}{l}\text { Non } \\
\text { connue }\end{array}$ & Non \\
\hline
\end{tabular}

*cps : comprimés

*PFC : plasma frais congelé $\quad *$ CGR : culot globulaire

Tableau 7 : Prise en charge de l'HPPI

Ce tableau nous résume la prise en charge hémorragique des patientes ayant accouché au centre d'étude.

Nous pouvons observer dans un premier temps que les utérotoniques utilisés à visée thérapeutique sont le Cytotec ${ }^{\circledR}$ et le Méthergin ${ }^{\circledR}$.

Le délai de la prise en charge initiale est rapide dès que le diagnostic d'hémorragie est posé. Par contre, lorsqu'un traitement chirurgical s'avère être nécessaire, le délai est plus long, avoisinant 
ainsi 1h. Cela s'explique par le manque de personnels mais aussi de la disponibilité du bloc opératoire. Aussi, le long délai de la suture de la déchirure cervicale est dû au fait que les trois salles de blocs opératoires étaient occupées ; les révisions du col se font au bloc opératoire.

En ce qui concerne le début de la transfusion des patientes, ces délais s'expliquent par le manque de ressources de la famille des patientes. Comme vu précédemment, les frais hospitaliers sont entièrement à la charge des familles. Ainsi, lorsqu'une ordonnance est donnée à la famille, il faut le temps que cette dernière rassemble l'argent nécessaire afin d'honorer la prescription. Un dosage du taux d'hémoglobine $(\mathrm{TH})$ a été réalisé pour la patiente ayant subit une hystérectomie d'hémostase : sa valeur était de $6,80 \mathrm{~g} / \mathrm{dl}$ après la première de culot globulaire et de plasma frais congelé. Il est à noté que cette patiente n'était pas anémiée à son admission (ses conjonctives étaient colorées).

\section{b. Caractéristiques et prise en charge des patientes transférées pour HPPI}

L'étude de la prise en charge des patientes transférées a été faite sur les dossiers obstétricaux des huit patientes concernées.

\begin{tabular}{lcc}
\multicolumn{1}{r}{ CRITERES } & Nombre (n) & \%(\% sur totalité des HPPI) \\
\hline Age & 6 & $75(42.9)$ \\
$<35$ ans & 2 & $25(14.3)$ \\
$\geq 35$ ans & & \\
Parité & 1 & $12.5(7.1)$ \\
-primipare & 4 & $50(28.6)$ \\
-multipare & 3 & $37.5(21.4)$ \\
-grande multipare & & 0 \\
Suivi de la grossesse & 0 & $12.5(7.1)$ \\
-pas de suivi & 1 & $62.5(35.7)$ \\
-peu de suivi & 5 & $25(14.3)$ \\
-suivi régulier & 2 & \\
-Absence de données &
\end{tabular}

\section{Tableau 8 : Caractéristiques des patientes transférées pour HPPI}

En ce qui concerne les patientes transférées pour une HPPI, 25\% étaient âgées de plus de 35 ans. Plus du tiers, étaient une grande multipare c'est-à-dire au moins 5 enfants. Mais $62.5 \%$ ont eu au moins 4 consultations prénatales.

Nous n'avons aucune donnée sur le déroulement du travail de ces patientes. 


\begin{tabular}{|c|c|c|c|c|c|c|c|}
\hline Patientes & $\begin{array}{l}\frac{\text { Cause }}{\text { HPPI }} \\
\underline{\underline{H}}\end{array}$ & $\begin{array}{l}\text { Délai de } \\
\text { transfert/ } \\
\text { Délai de PEC }\end{array}$ & $\begin{array}{l}\text { Traitement } \\
\text { initial }\end{array}$ & Utérotonique & $\begin{array}{l}\text { Délai } \\
\text { transfusion / } \\
\text { Nombre de } \\
\text { culots }\end{array}$ & $\underline{\text { Pertes }}$ & $\begin{array}{l}\frac{\text { Traitement }}{\text { chirurgical }} \\
\text { Lautres } \\
\text { examens }\end{array}$ \\
\hline $\begin{array}{l}28 \text { ans, } \\
1 P^{*} \\
\text { Suivi } \\
\text { régulier }\end{array}$ & $\begin{array}{l}\text { Suspicion } \\
\text { déchirure } \\
\text { du col }\end{array}$ & $\begin{array}{c}\text { Délai de PEC: } \\
1 \mathrm{~h} 30\end{array}$ & Non connu & Ocytocine & $\begin{array}{l}\text { Non } \\
\text { transfusée }\end{array}$ & $\begin{array}{l}\text { Non } \\
\text { connues }\end{array}$ & $\begin{array}{c}\text { Non } \\
\mathrm{TH}^{*}=10.8 \mathrm{~g} / \mathrm{dl}\end{array}$ \\
\hline $\begin{array}{l}30 \text { ans, } 2 P \\
\text { peu de } \\
\text { suivi } \\
\text { NN *mort- } \\
\text { né }\end{array}$ & $\begin{array}{l}\text { Rétention } \\
\text { placentaire }\end{array}$ & $\begin{array}{c}\text { Délai de } \\
\text { transfert : } \\
1 \mathrm{~h} 20 \\
\text { Délai de PEC : } \\
\text { 40minutes }\end{array}$ & $\begin{array}{c}\text { Révision } \\
\text { utérine(RU), } \\
\text { massage } \\
\text { utérin }\end{array}$ & $\begin{array}{l}\text { Ocytocine } \\
5 \text { cps de } \\
\text { Cytotec } \AA\end{array}$ & $\begin{array}{c}\text { Non } \\
\text { transfusée }\end{array}$ & $\begin{array}{c}\text { Non } \\
\text { connues }\end{array}$ & Non \\
\hline $\begin{array}{l}26 \text { ans, } 2 p \\
\text { suivi } \\
\text { régulier }\end{array}$ & $\begin{array}{l}\text { Rétention } \\
\text { placentaire }\end{array}$ & $\begin{array}{c}\text { Délai } \\
\text { transfert : } \\
7 \text { h00 } \\
\text { PEC immédiate }\end{array}$ & $\begin{array}{l}\text { Idem + } \\
\text { Remplissage } \\
\text { vasculaire } \\
\text { (Gélofusine }{ }^{\circledR} \text { ) }\end{array}$ & Ocytocine & $\begin{array}{l}3 \mathrm{~h} 00 \\
2 \mathrm{CGR}\end{array}$ & $\begin{array}{c}\text { Non } \\
\text { connues }\end{array}$ & Non \\
\hline $\begin{array}{l}25 \text { ans, } 2 p \\
\text { Suivi } \\
\text { régulier }\end{array}$ & $\begin{array}{l}\text { Rétention } \\
\text { placentaire }\end{array}$ & $\begin{array}{c}\text { Délai de } \\
\text { transfert : } \\
11 \text { h00 } \\
\text { Délai de PEC: } \\
\text { immédiat }\end{array}$ & $\begin{array}{l}\text { RU, massage } \\
\text { utérin, } \\
\text { sondage, }\end{array}$ & $\begin{array}{l}\text { Ocytocine } \\
5 \text { cps } \\
\text { Cytotec } ®\end{array}$ & $\begin{array}{l}1 \mathrm{~h} 30 \\
1 \mathrm{CGR}\end{array}$ & $\begin{array}{c}\text { Non } \\
\text { connues }\end{array}$ & Non \\
\hline $\begin{array}{l}39 \text { ans, } \\
5 p \\
\text { Suivi } \\
\text { régulier }\end{array}$ & $\begin{array}{l}\text { Rétention } \\
\text { placentaire }\end{array}$ & $\begin{array}{c}\text { Délai de } \\
\text { transfert : } 3 \mathrm{~h} \\
\text { PEC immédiate }\end{array}$ & idem & Ocytocine & $\begin{array}{l}08 \mathrm{~h} 00 \\
1 \mathrm{CGR}\end{array}$ & $\begin{array}{c}\text { Non } \\
\text { connues }\end{array}$ & Non \\
\hline $\begin{array}{l}34 \text { ans, } 4 \mathrm{p} \\
\text { suivi } \\
\text { régulier } \\
\text { DTT *: } \\
4 \mathrm{~h} 20 \\
\end{array}$ & $\begin{array}{l}\text { Rétention } \\
\text { placentaire }\end{array}$ & $\begin{array}{c}\text { Délai } \\
\text { transfert : } \\
2 \mathrm{~h} 20 \\
\text { Délai PEC: } \\
\text { immédiate }\end{array}$ & $\begin{array}{c}\text { Idem + } \\
\text { Remplissage } \\
\text { vasculaire } \\
\text { (Gélofusine }\left({ }^{\circledR}\right)\end{array}$ & $\begin{array}{l}\text { Ocytocine } \\
5 \text { cps de } \\
\text { Cytotec } ®\end{array}$ & $\begin{array}{l}50 \text { minutes } \\
2 C G R\end{array}$ & $\begin{array}{l}\text { Non } \\
\text { connues }\end{array}$ & Non \\
\hline $\begin{array}{l}40 \text { ans, 3p } \\
\text { Patiente } \\
\text { arrivée en } \\
\text { état de } \\
\text { choc }\end{array}$ & $\begin{array}{l}\text { Rétention } \\
\text { placentaire }\end{array}$ & $\begin{array}{c}\text { Délai de } \\
\text { transfert : } \\
\text { non connu } \\
\text { Délai de PEC: } \\
\text { immédiat }\end{array}$ & Idem & $\begin{array}{l}\text { Ocytocine } \\
5 \text { cps de } \\
\text { Cytotec } \AA\end{array}$ & $\begin{array}{c}40 \text { minutes } \\
2 \text { CGR et } 1 \\
\text { PFC }\end{array}$ & $\begin{array}{l}\text { Non } \\
\text { connues }\end{array}$ & Non \\
\hline $\begin{array}{l}25 \text { ans, } 4 p \\
\text { HTA } \\
\text { gravidique } \\
\text { Grossesse } \\
\text { gémellaire }\end{array}$ & $\begin{array}{l}\text { Rétention } \\
\text { placentaire }\end{array}$ & \begin{tabular}{l}
\multicolumn{1}{c}{ Délai de } \\
transfert : $4 \mathrm{~h}$ \\
Délai de PEC: \\
immédiat
\end{tabular} & $\begin{array}{l}\text { RU, massage, } \\
\quad \text { sondage } \\
\text { Remplissage } \\
\text { vasculaire } \\
\text { (Plasmion } ®)\end{array}$ & $\begin{array}{l}\text { Ocytocine } \\
5 \text { cps de } \\
\text { cytotec } \AA\end{array}$ & $\begin{array}{l}5 \text { h00 } \\
2 \text { CGR }\end{array}$ & $\begin{array}{c}\text { Non } \\
\text { connues }\end{array}$ & Non \\
\hline
\end{tabular}




\begin{tabular}{|c|c|c|}
\hline CRITERES & Nombre (n) & $\%$ (\% sur le total d'HPPI) \\
\hline \multicolumn{3}{|l|}{ Causes de l'HPPI } \\
\hline -Rétention placentaire & 7 & $87.5(50)$ \\
\hline -autre & 1 & $12.5(7.1)$ \\
\hline \multicolumn{3}{|l|}{ Délai de transfert } \\
\hline$-<2 \mathrm{~h}$ & 2 & $25(14.3)$ \\
\hline$-2 h-4 h$ & 3 & $37.5(21.4)$ \\
\hline$->4 \mathrm{H}$ & 2 & $25(14.3)$ \\
\hline$-N S P$ & 1 & $12.5(7.1)$ \\
\hline \multicolumn{3}{|l|}{ Utérotonique } \\
\hline -ocytocine seul & 3 & $37.5(21.3)$ \\
\hline -ocytocine + Cytotec $®$ & 5 & $62.5(35.7)$ \\
\hline \multicolumn{3}{|l|}{ Transfusion } \\
\hline- Non & 2 & $25(14.3)$ \\
\hline -oui & 6 & 75 (42.9) \\
\hline \multicolumn{3}{|l|}{ Délai de transfusion } \\
\hline$-<1 h$ & 2 & $33.3(14.3)$ \\
\hline$-1 h-3 h$ & 2 & $33.3(14.3)$ \\
\hline$->3 h$ & 2 & $33.3(14.3)$ \\
\hline
\end{tabular}

$\underline{\text { Tableau } 10}$ : prise en charge des patientes transférées

Quatre-vingt-sept pour cent des HPPI ont pour étiologie une rétention placentaire.

Une des patientes a été transférée au centre pour une suspicion de déchirure cervicale mais les informations contenues dans le dossier ne nous informent pas si une révision du col sous valves a pu confirmer la suspicion. Mais à l'admission de la patiente il n'y avait «pas de saignements actifs visualisés à l'arrivée ».

Le délai de transfert correspond au temps écoulé entre l'heure d'accouchement et l'heure d'arrivée de la patiente au CHU-SO.

Les délais de transfert peuvent s'expliquer surtout par le manque de moyens des patientes. En effet, souvent, les patientes arrivent par leurs propres moyens.

A leur arrivée, les patientes ont toutes bénéficié d'une prise en charge immédiate. Cette prise en charge consiste en la réalisation d'une révision utérine, d'un massage utérin et souvent d'un sondage urinaire. Une voie veineuse périphérique est aussi posée avant l'arrivée du médecin. $62.5 \%$ ont reçu une association ocytocine-misoprostol et $75 \%$ ont été transfusées. Les patientes que l'on peut considérer comme ayant surement eu une hémorragie grave (transfusion d'au moins 2 culots globulaires) ont eu un remplissage vasculaire par Plasmion® ou Gélofusine®. 
On peut mettre en relation le délai de transfert et la proportion des patientes transfusées à leur arrivée.

\begin{tabular}{lccc}
$\begin{array}{l}\text { Délai de } \\
\text { transfert }\end{array}$ & $\begin{array}{c}\text { Non transfusée } \\
\text { n }\end{array}$ & Transfusion & Total \\
\hline$<2 \mathrm{~h}$ & 2 & 0 & 2 \\
\hline $2 \mathrm{~h}-4 \mathrm{~h}$ & 0 & 3 & 3 \\
$>4 \mathrm{~h}$ & 0 & 2 & 2 \\
Absence de données & 0 & 1 & 1 \\
Total & 2 & 6 & 8 \\
\hline
\end{tabular}

\section{Tableau 11: Transfusions sanguines en fonction du délai de transfert des patientes}

Ainsi, ce sont majoritairement les patientes transférées plus de 2 heures après leur accouchement qui ont été transfusées.

Parmi les patientes ayant présenté une HPPI durant la période d'étude, $71.5 \%$ étaient âgées de moins de 35 ans et avaient un suivi régulier de leur grossesse. A l'admission, cinq (soit 35,7\%) présentaient un facteur de risque d'HPPI (autre que l'âge, la grande parité et le suivi de grossesse) : placenta prævia, distension utérine, utérus polymyomateux.

La rétention placentaire constitue au total 50\% des causes d'HPPI, suivie de l'atonie utérine et de la déchirure cervicale à $14.3 \%$ chacun. Dans $21.4 \%$ des cas, la cause d'HPPI n'était pas mentionnée dans les dossiers. La prise en charge des HPPI au CHU-SO ne diffère pas du fait que la patiente y a accouchée ou y a été transférée après l'accouchement. Les patientes sont prises en charge dès que le diagnostic est posé ou dès leur arrivée pour celles qui ont accouché ailleurs. Elles ont toutes bénéficié d'une révision utérine suivie d'un massage utérin et de la pose d'une voie veineuse périphérique avec une perfusion lente d'ocytocine. Soixante-quatre pourcent ont reçu du Cytotec ${ }^{\circledR}$ en plus de l'ocytocine et $14 \%$ une injection de $1 \mathrm{mg}$ de Méthergin ${ }^{\circledR}$.

Au total, $71.4 \%$ des patientes ont été transfusées, $14 \%$ ont eu une chirurgie conservatrice (ligature vasculaire) et 7\% (soit 1 patiente) ont eu une hystérectomie d'hémostase. L'hystérectomie fut secondaire à la ligature vasculaire. Pendant la durée d'étude, aucune patiente n'est décédée des suites d'une hémorragie du post-partum immédiat. 


\section{Résultats des entretiens}

Le tableau ci-après détaille les réponses données par les professionnels lors des entretiens.

Six professionnels de santé ont été interrogés : trois sages-femmes et trois obstétriciens. Ce sont tous des accoucheurs qualifiés. Le diplôme de sage-femme est délivré par l'Ecole nationale des sages-femmes du Togo après validation des trois années de formation. Le statut professionnel de gynécologue-obstétricien s'acquiert après 8 ans d'études médicales générales et quatre ans de préparation du Certificat d'études spécialisées de gynécologie-obstétrique.

Les causes et les facteurs de risques d'HPPI les plus énoncées par l'ensemble des professionnels interrogés sont le placenta prævia, la surdistension utérine et un travail obstétrical trop long. La pré-éclampsie a été citée par 5 professionnels. Deux-tiers des obstétriciens estiment que l'anémie et un trouble de la coagulation peuvent être à l'origine d'une hémorragie, alors qu'aucune des sagesfemmes n'a évoqué ces possibilités. Seule la moitié du personnel interrogée cite l'hématome rétro placentaire (HRP) et un décollement placentaire (DPPNI) comme pouvant causer une HPPI. Enfin, seul un obstétricien évoque les causes infectieuses et l' «usage intempestif » de l'ocytocine pendant le travail. Donc, les différents facteurs de risques d'HPPI sont relativement bien connus par le personnel soignant malgré une certaine hétérogénéité des réponses entre les sages-femmes et les médecins.

Les moyens de prévention les plus cités sont le massage utérin et le misoprostol en intra-rectal. Nous pouvons remarquer que la totalité des personnes interrogées connaissent et pratique la gestion active de la troisième phase de l'accouchement (GATPA) pour chaque naissance par voie basse.

Dans le cadre de la gestion de l'HPPI, en plus des actes techniques tels que la révision utérine, le massage utérin et le sondage urinaire, 2/3 des médecins estiment que la sage-femme doit avoir évalué les pertes sanguines de la patiente avant de les appeler. Or, l'estimation des pertes n'est citée par aucune sage-femme dans la gestion de l'hémorragie.

En ce qui concerne le ressenti de ces professionnels sur les taux élevés d'HPPI, plusieurs causes ont été évoquées notamment le manque de suivi des patientes pendant la grossesse et le manque de matériels. Ces deux importantes causes sont la conséquence d'une (quasi) absence de couverture sociale et l'absence de drogues d'urgence à la disposition du personnel. Une sage-femme souligne l'«incompétence du personnel dans les centres médico-sociaux et autres centres de soins». En 
effet, au Togo et plus particulièrement à Lomé, il existe de nombreuses maisons d'accouchements. Or, il n'existe aucune surveillance, par l'Etat, des compétences des personnes dirigeants ces maisons d'accouchements. Ainsi donc, à Lomé pour citer un des obstétriciens «n'importe qui peut se lever un beau matin et ouvrir une maison d'accouchement » sans être inquiété par aucune Autorité politique ni judiciaire.

Enfin, l'ensemble du personnel soignant estime qu'il est indispensable d'accroître la prévention. Pour ce, il est nécessaire de sensibiliser la population togolaise à la nécessité d'un suivi régulier de la grossesse et d'un accouchement en milieu hospitalier mais aussi d'augmenter de l'effectif du personnel soignant et renforcer la formation de ce dernier. 


\begin{tabular}{|c|c|c|}
\hline & SAGE-FEMME & OBSTETRICIEN \\
\hline DUREE D'ACTIVITES & & \\
\hline $\begin{array}{l}<\text { 3ans } \\
\text { 3-6ans } \\
\geq 7 \text { ans }\end{array}$ & $\begin{array}{l}1 \\
1 \\
1\end{array}$ & $\begin{array}{l}0 \\
1 \\
2\end{array}$ \\
\hline CONNAISSANCES & & \\
\hline $\begin{array}{l}\text { DEFINITION HPPI } \\
\text { VB }: 500 \mathrm{cc} \text { dans les } 24 \mathrm{~h} \\
\text { VH }: 1000 \mathrm{cc} \text { en } 24 \mathrm{~h}\end{array}$ & $\begin{array}{l}2^{3} \\
2\end{array}$ & $\begin{array}{l}3 \\
2\end{array}$ \\
\hline $\begin{array}{l}\text { CAUSES HPPI } \\
\text {-Anémie } \\
\text {-Déchirure des parties } \\
\text { molles } \\
\text {-DPPNI } \\
\text {-HRP } \\
\text {-Infection ovulaire } \\
\text {-Placenta prævia } \\
\text {-Pré-éclampsie } \\
\text {-Rétention placentaire } \\
\text { (atonie utérine) } \\
\text {-Rupture utérine } \\
\text {-Surdistension utérine } \\
\text {-Travail long } \\
\text {-Travail rapide } \\
\text {-Troubles de la coagulation } \\
\text {-Usage d'ocytocine } \\
\text { pendant le travail }\end{array}$ & $\begin{array}{l}0 \\
1 \\
2 \\
0 \\
0 \\
3 \\
3 \\
2 \\
0 \\
3 \\
3 \\
1 \\
0 \\
0\end{array}$ & $\begin{array}{l}2 \\
2 \\
1 \\
3 \\
1 \\
3 \\
2 \\
3 \\
1 \\
3 \\
3 \\
1 \\
2 \\
1\end{array}$ \\
\hline $\begin{array}{l}\text { PREVENTION SI } \\
\text { FACTEUR DE RISQUE } \\
\text {-Commande de sang } \\
\text {-Massage utérin } \\
\text {-Misoprostol } \\
\text {-Révision utérine }\end{array}$ & $\begin{array}{l}0 \\
3 \\
3 \\
0\end{array}$ & $\begin{array}{l}1 \\
2 \\
3 \\
1\end{array}$ \\
\hline
\end{tabular}

3 « HPPI = 500cc dans les $2 \mathrm{~h}$ suivant accouchement. Idem pour la césarienne » 


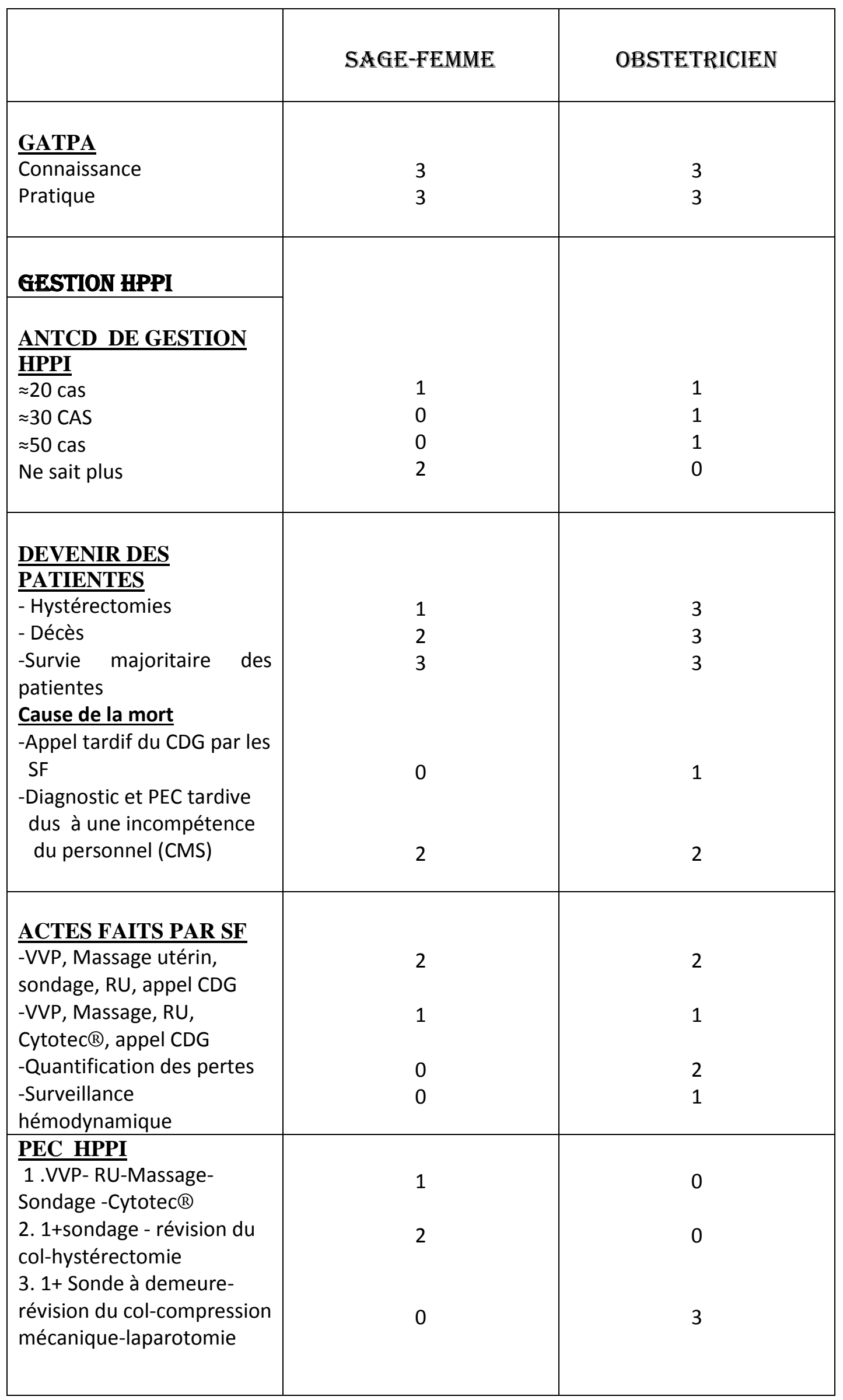




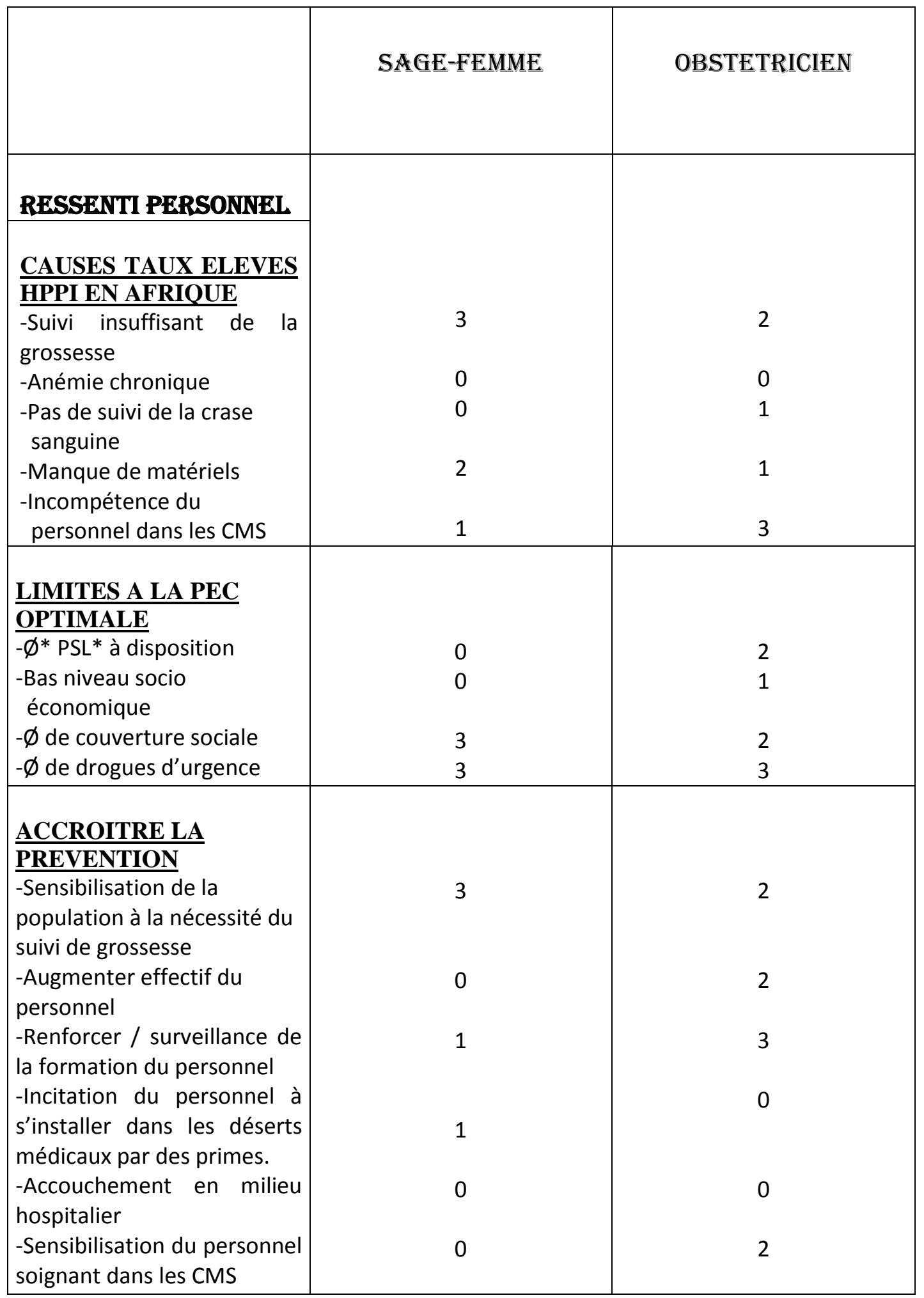

*GATPA : Gestion active de la troisième phase de l'accouchement $* \varnothing$ : Absence

* $\underline{\mathrm{CMS}}$ : Centre médico-social ${ }^{*} \underline{\mathrm{CDG}}$ : Chef de garde ${ }^{*} \underline{\mathrm{VP}}$ : Voie veineuse périphérique

*PSL : Produits sanguins labiles

Tableau 12: Résultats de l'entretien avec les professionnels 


\section{Quatrième partie: Discussion}

L'hémorragie du post-partum immédiat est la première cause de mortalité chez les jeunes femmes dans les pays en voie de développement. Selon l'OMS, la probabilité qu'une jeune femme décèdera un jour d'une cause liée à la grossesse ou à l'accouchement est de 1/150 contre 1/3800 dans les pays développés.

La réduction de la mortalité par HPPI passe par la mise en application des recommandations internationales, concernant la prévention et la prise en charge de l'HPPI.

Nous avons cherché à évaluer si la prévention et la prise en charge de l'HPPI au CHU Sylvanus Olympio étaient conformes aux recommandations de l'OMS et de la FIGO, et quelle la formation du personnel à la PEC de l'HPPI.

\section{Prévention de l'HPPI au CHU-SO}

L'analyse des recueils de donnés sur dossiers et à partir de l'observation met en évidence une certaine conformité des pratiques togolaises avec les recommandations internationales

\section{Suivi de la grossesse}

Conformément aux recommandations de l'OMS, $62.7 \%$ des patientes ont eu au moins 4 consultations prénatales durant leur grossesse. Ce chiffre reste en deçà des objectifs attendus, à savoir que toute femme enceinte ait au moins 4 CPN durant la grossesse.

De l'entretien avec les professionnels, a émergé la problématique des «cliniques sauvages » : les maisons d'accouchements qui sont gérées par un personnel non qualifié. En 2006, plus de 800 cliniques sauvages ont été dénombrées sur l'ensemble du territoire togolais, dont une grande majorité à Lomé. Au mieux ces personnes sont issues du milieu paramédical (infirmiers, aides opératoires et autres). Dans des cas extrêmes, elles n'ont eu aucune formation dans le domaine de 
la santé ; ce sont des couturiers, mécaniciens, agriculteurs et autres métiers n'ayant aucun lien avec le domaine de la santé. Depuis 2005, du fait d'un grand nombre de décès sans explications dans ces maisons, l'Etat tente d'éradiquer ce phénomène sans résultats concrets. En effet les cliniques sauvages continuent de croître pour une raison principale : la population considère ces lieux de « soins » comme un service de proximité. Du fait du désert médical dans les régions reculées, du manque de personnel dans les hôpitaux publics et donc d'une attente très longue lors des consultations, une partie de la population togolaise considère ces maisons de naissance comme une alternative aux hôpitaux et cliniques ; ne prenant pas conscience du danger encourut. Si donc l'Etat n'est pas en mesure d'éradiquer totalement ce phénomène dangereux à la source, il est important, de prévenir la population sur les dangers de ces pratiques. Ainsi en 2013, une campagne de sensibilisation de la population a été lancée via notamment un spot publicitaire diffusé à la télévision dans les principaux dialectes, qui met l'accent sur la nécessité d'un suivi obstétrical par un personnel qualifié pendant la grossesse.

\section{Prévention de l'HPPI au CHU-SO}

Comme dans les articles publiés, nous constatons que la population étudiée est constituée majoritairement de femmes à bas risque d'HPPI (patientes jeunes ayant un utérus sain et entrant en travail spontanément).

\section{a. Pendant le travail}

Au cours de cette étude, nous avons relevé peu d'utilisation d'ocytocine. En effet le taux de patientes ayant reçu de l'ocytocine pendant le travail était de $11.7 \%$. Or, il est connu que l'usage de l'ocytocine pendant le travail est un facteur de risque important d'HPPI. (29)

En revanche, nous avons été surpris du taux de césariennes de $38.4 \%$ portant aussi bien sur les césariennes avant travail que les césariennes pendant le travail. Ceci d'autant plus surprenant qu'il n'existe pas de monitorage continu du rythme cardiaque fotal au cours du travail : la surveillance fœtale reposait sur l'auscultation intermittente à l'aide d'un stéthoscope de pinard et la couleur du liquide amniotique. En effet, les césariennes sont un facteur de risque bien identifié de l'HPPI ; notamment pour celles en cours de travail.

Les efforts gouvernementaux se manifestent aussi dans la gestion du travail. En 2011, une augmentation des financements des dépenses de santé (8\% du PIB en 2011 contre $7.2 \%$ en 2009) a permis entre autres la mise en place des kits d'accouchement et de césarienne. Le prix 
initial du kit de césarienne était de 60000 FCFA (91€60). Mais depuis mai 2011, devant le taux de mortalité maternelle et infantile par manque d'accès à une césarienne en urgence, l'Etat prend en charge près de $90 \%$ des frais de césariennes. Les patientes ne déboursent plus que 10000 FCFA $(15 € 30)$ : l'Etat prenant en charge les 50000FCFA $(76 € 30)$ restants ainsi que les autres frais pouvant découler de la césarienne à hauteur de 20000FCFA (30€53). Cela s'inscrit dans la lutte contre la mortalité maternelle et infantile, la CARMMA (Campagne pour l'Accélération de la Réduction de la Mortalité Maternelle en Afrique) initiée en septembre 2010 au Togo(30).

\section{b. Lors de l'accouchement et de la délivrance}

Concernant la prise en charge de la délivrance, en cas de voie basse, nous avons obtenu des pratiques conformes aux recommandations de l'OMS. Ainsi, la GATPA a été réalisée de façon quasi-systématique dans plus de $95 \%$. Les cas de réalisation systématique d'une délivrance artificielle étaient motivés par l'existence d'une mort fœtale in utéro, dont le sur risque hémorragique est bien connu en Afrique.

Mais il est à noter, que l'hôpital dispose d'un stock d'ocytocine permettant l'administration dans tous les cas, alors que la presque totalité des consommables est à la charge des patients pour presque tous les soins.

\section{c. Dans le post-partum immédiat}

La surveillance du post-partum est aussi conforme. En effet, d'après les données disponibles, la surveillance du post-partum dure le plus souvent au moins deux heures. L'administration de l'ocytocine a été faite dans $100 \%$ des cas. Mais durant cette période d'observation, aucune ampoule de Syntocinon ${ }^{\circledR}$ n'était conservée au frais entre le moment d'achat des kits d'accouchements par la famille des patientes et l'utilisation par la sage-femme. Or, pour une efficacité optimale, l'ocytocine de synthèse doit être conservé à une température n'excédent pas $+8^{\circ} \mathrm{C}$.

Concernant les femmes à risque accru d'HPPI, le protocole de prévention consistait en l'administration systématique de 5 comprimés de misoprostol en intra rectale. La non conservation des ampoules de Syntocinon ${ }^{\circledR}$ au réfrigérateur, diminue donc l'efficacité de ce dernier. Son association avec le Cytotec $®$ chez ces patientes, peut permettre ainsi de palier à la faible efficacité du Syntocinon ${ }^{\circledR}$ conservé à température ambiante.

Ce protocole n'est pas conforme aux recommandations de l'OMS ni de la FIGO qui préconisent l'ocytocine en prévention chaque fois qu'elle est disponible car il s'agit du traitement de référence. Néanmoins, le misoprostol est un utérotonique dont l'efficacité dans la prévention et la prise en charge de l'HPP est reconnu. 


\section{Prise en charge de l'HPPI}

La fréquence de l'hémorragie du post-partum retrouvée lors de cette étude est de 4,7\%.Ce résultat est conforme aux fréquences retrouvées dans la littérature : 5\%.

Le recueil de données concernant la prise en charge de l'HPPI a été le plus difficile. En effet, un seul cas d'HPPI a été observé par nous-mêmes. De plus, le recueil comportait de nombreuses données manquantes.

\section{a. Les connaissances des professionnels}

Les connaissances sur la définition et les causes d'HPPI sont assez hétérogènes. En revanche, la connaissance de la prévention et notamment de la GATPA est universelle.

La prise en charge de l'HPPI est plus mal connue et hétérogène chez les sages-femmes qui « passent la main » lorsque l'hémorragie nécessite une prise en charge autre que la révision utérine et l'usage du Syntocinon®.

\section{b. Prise en charge initiale}

Dans le cas observé, certains actes et leur chronologie sont conformes aux recommandations, c'està-dire, la révision utérine suivie d'un sondage vésical, massage utérin par la sage-femme, puis l'appel de l'obstétricien qui a prit la décision d'une révision du col sous valves.

D'autres gestes ont été tardifs ou manquants. Ainsi, la révision du col n'a lieu qu'après 1 h20 minutes après le début de l'HPPI, le Syntocinon ${ }^{\circledR}$ n'a pas été administré à la phase initiale de l’hémorragie. Enfin, la quantification des pertes sanguines a été visuelle.

Dans les cas analysés sur dossiers, nous avons pu observer que 2/3 des patientes n'étaient pas suivies au CHU-SO et y ont été référées qu'au moment de l'HPPI. Ainsi, nous n'avons pas d'informations sur les évènements antérieurs à leur hospitalisation au CHU.

Le Cytotec ${ }^{\circledR}$ et le Méthergin ${ }^{\circledR}$ constituaient les traitements de l'HPPI. Malgré les effets secondaires de l'ergométrine décrits dans la première partie, cette substance est toujours utilisée au Togo. Ne disposant dans d'autres moyens intermédiaires entre le misoprostol et la ligature vasculaire, les professionnels ayant pesée la balance bénéfice-risque préfèrent l'utiliser de manière ponctuelle et en respectant les contre-indications notamment l'hypertension artérielle. 
Pour ce qui est de la disponibilité du sang pour les transfusions, l'absence de disponibilité de produits sanguins au sein du service, le coût des examens pour réaliser une carte de groupe sanguin et le fait que l'achat des culots globulaires soient à la charge des familles sont les causes des retards à la transfusion que nous avons pu observer voire l'absence de transfusion.

\section{c. Cas particuliers des patientes transférées : rôle des centres de soins primaires}

Parmi les femmes transférées pour HPPI, $26.7 \%$ auraient pu être dirigées plus précocement durant leur grossesse à un hôpital plus adapté. Il s'agit des patientes présentant un placenta prævia ou un utérus cicatriciel. Se pose donc la question de la gestion et de la prise en charge des patientes dans les centres de soins primaires (CMS, dispensaires, maisons d'accouchements...). De plus, les causes d'HPPI des patientes transférées nous montrent que dans ces centres de soins primaires, la compétence du personnel soignant n'est pas adaptée. En effet, $87.9 \%$ des patientes transférées pour HPPI avaient une rétention placentaire. Or, le diagnostic de la rétention placentaire est facile et le traitement est simple : la révision utérine. De tous les accouchements que nous avons pu observer, aucun examen du placenta n'a été réalisé au centre de référence et la probabilité que cet examen recommandé par l'OMS soit réalisé dans les centres annexes est faible.

Enfin, le délai de transfert de ces patientes ayant une simple rétention utérine constitue une perte de chance et augmente les risques de conséquences de l'HPPI, puisque la prise en charge d'une hémorragie doit se faire le plus rapidement possible et le plus proche du possible du lieu d'accouchement. Cela se confirme par le taux de patientes ayant nécessité une transfusion : plus le délai de prise en charge est tardif plus la patiente a de risque d'être transfusée.

\section{Forces et limites de l'étude}

\section{Les limites}

\section{a. Une étude monocentrique}

Cette étude n'a été réalisée qu'au CHU Sylvanus Olympio. Toutes les pratiques décrites sont celles observées dans un seul centre. Même si celui constitue le centre de référence sur l'ensemble du territoire national, la prise en charge des patientes est surement différente dans d'autres hôpitaux 
que ce soit dans le secteur privé ou dans les CMS. De plus, nous ne savons pas quelle a été la prise en charge antérieure des patientes transférées

\section{b. Qualité des variables recueillies}

Dans les dossiers obstétricaux, de nombreuses données étaient manquantes à chaque niveau de l'étude. Cette absence de données concerne environ $4 \%$ des dossiers et concerne aussi bien le suivi de la grossesse, que le déroulement du travail, de l'accouchement et de la surveillance du postpartum immédiat. Ce manque d'informations concerne autant les patientes ayant accouchées au CHU-SO que celles y ayant été transférées pour HPPI dont nous n'avons aucune information concernant le déroulement de la grossesse, du travail, ni de l'accouchement.

L'analyse de la surveillance du post-partum immédiat, fut limitée par un manque de données notamment pour les accouchements par voie basse. Mais durant le stage optionnel, nous avons pu observer qu'un examen de sortie de la salle des accouchées est réalisé par la sage-femme ou la garde-malade. Il consiste la plupart du temps à une vérification du globe utérin et de la garniture (constituée à l'aide d'un pagne appartenant à la femme) de la patiente avant la montée en suites de couches. Ainsi donc, l'absence de données sur la surveillance du post-partum des accouchées par voie basse ne signifie pas que cette surveillance est inexistante mais par manque de temps, et/ou d'habitude les sages-femmes ne font pas apparaitre cet élément dans les dossiers obstétricaux.

La sous-estimation des pertes sanguines est mise en évidence dans le cas de deux patientes ayant accouchées au CHU-SO mais qui n'aurait perdues que $150 \mathrm{cc}$ de sang alors que l'une a été transfusée d'une poche de plasma frais congelé et d'un culot globulaire, tandis que la deuxième patiente a subit une ligature vasculaire. En effet au CHU-SO, il n'existe aucune méthode de quantification des pertes sanguines. Seules les pertes des césariennes sont estimées et cette estimation est visuelle. Ainsi donc, il est probable que des patientes aient perdu plus de $500 \mathrm{ml}$ suite à leur accouchement mais ayant bien toléré cette perte sanguine, elles n'ont pas été transfusées et n'ont donc pas été incluses dans les cas d'HPPI relevés.

Enfin, les limites de l'évaluation de la formation des professionnels sont le faible nombre de personnels interrogés. 


\section{Les forces de l'étude}

\section{a. Une enquête de terrain}

La réalisation de cette étude a nécessité une investigation sur place. Un voyage entrepris dans le cadre de mon stage optionnel, afin d'étudier les pratiques obstétricales à travers l'étude des dossiers et l'observation et ce grâce à mes connaissances en tant qu'étudiante sage-femme.

\section{b. Intégration au sein de l'équipe obstétricale}

La réalisation de ce travail a aussi été facilitée par l'accueil chaleureux de l'ensemble de l'équipe obstétricale : l'accès à l'ensemble des dossiers médicaux ainsi qu'aux différents services de la maternité. Enfin, malgré la charge de travail importante, les professionnels interrogés ont pu consacrer le temps nécessaire à la réalisation des entretiens sans aucune réticence.

\section{Propositions d'amélioration}

Ce travail permet d'identifier des difficultés propres à chaque étape de prévention et de traitement de l'HPPI :

- En amont de l'accouchement

- Pendant le travail

- Dans le post-partum immédiat

$\bullet$

\section{L'accès aux soins pendant la grossesse}

Selon l'UNICEF, 72\% des femmes togolaises enceintes ont réalisé au moins une visite et $55 \%$ au moins 4 consultations prénatales entre 2007-2012 conformément aux recommandations de l'OMS. Dans notre étude, nous avons retrouvé un taux de 62.7\%. Comme vu dans l'introduction, une consultation prénatale (CPN) au CHU-SO coûte 1500 FCFA soit 2,30€; ainsi la somme de 4 CPN revient à 6000FCFA à chaque patiente soit 9,20€. En 2011, il eut 195000 naissances au Togo. Le coût, à l'échelle de l'ensemble du pays d'un suivi prénatal systématique s'élèverait ainsi à $1794000 €$ par an. On peut se demander s'il ne serait pas possible pour l'Etat togolais de rendre 
ces 4 CPN gratuites pour toutes les femmes enceintes ou du moins d'en réduire le coût comme ce fut le cas pour le kit de césarienne.

Toujours selon l'UNICEF, seuls $67 \%$ des accouchements ont eu lieu dans une institution légale dont $91 \%$ des naissances ont été assistées par un personnel soignant qualifié en milieu urbain contre $43 \%$ en milieu rural. La disparité entre la ville et les campagnes est importante. Au Togo, l'effectif du personnel soignant qualifié est peu élevé et concentré dans la capitale. En effet, il y a seulement 1 médecin pour 12470 habitants et 1 sage-femme pour 9330 habitants, sachant que près de la moitié de ces professionnels est concentrée à Lomé.

Pour réduire cette disparité entre les milieux urbains et ruraux, les accoucheuses traditionnelles pourraient être formées à l'obstétrique (les techniques d'accouchement et les principales complications de la grossesse, de l'accouchement et du post-partum), et devenir ainsi des sagesfemmes qualifiées ou comme l'a proposé une des sages-femmes, une motivation économique pourrait permettre d'homogénéiser la répartition médicale sur l'ensemble du territoire togolais : proposer des avantages aux personnels soignants qui accepteraient de quitter la vile pour les régions reculées de l'intérieur du pays : augmentation salariale, une habitation, un matériel médical neuf.

Ainsi, l'augmentation du nombre de personnel qualifié, l'homogénéisation des soins sur le territoire et la gratuité des CPN peuvent permettre aux femmes togolaises de ne plus se tourner vers les personnes exerçants illégalement la profession de sage-femme ou celle de gynécologueobstétricien. Ceci réduirait ainsi les risques de complications de la grossesse, de l'accouchement et du post-partum notamment l'HPPI.

Enfin, les efforts gouvernementaux notamment sur l'information de la population sont à poursuivre et à développer, en mettant par exemple en place des réunions d'informations dans les quartiers et /ou dans les marchés afin de sensibiliser aussi les femmes qui n’ont pas accès aux médias télévisés.

\section{Pendant le travail}

Au sein du CHU-SO, le taux de césarienne relevé est bien plus élevé que celui de la France. Même si l'on observe une augmentation mondiale du taux de césarienne qui participe à l'augmentation du risque accru d'HPPI, cela pourrait faire l'objet d'une analyse au sein de l'équipe du CHU-SO.

En dehors du CHU-SO, du fait de nombreux accouchements dans les centres de proximité, il est indispensable d'améliorer la prise en charge de l'HPPI notamment par des formations à la pratique 
de la délivrance artificielle et de la révision utérine, ainsi qu'à l'utilisation de l'ocytocine : l'HPPI doit se traiter au plus proche du lieu d'accouchement afin d'éviter les retards de prise en charge.

\section{Le post-partum immédiat}

\section{a. La prévention de l'HPPI}

Afin de garantir, l'efficacité de l'ocytocine il est nécessaire que les ampoules (que ce soit le stock disponible auprès des sages-femmes ou le contenu des kits d'accouchements) de Syntocinon ${ }^{\circledR}$ soient conservées au réfrigérateur.

La première cause d'HPPI retrouvée étant la rétention placentaire, il est désormais important que l'ensemble des professionnels du CHU et des autres centres de santé de l'ensemble du territoire togolais soit formé et sensibilisé à la nécessité de la réalisation de l'examen du placenta.

\section{b. Prise en charge}

Concernant la prise en charge de l'HPPI, il s'agira de mettre en place des moyens afin de limiter les délais d'utilisation d'utérotonique et de la révision utérine. C'est-à-dire agir soit sur le délai de transfert des patientes vers le $\mathrm{CHU}$ ou au mieux améliorer cette prise en charge initiale sur le lieu de l'accouchement. Les délais de la prise en charge chirurgicale lorsque cette dernière est nécessaire doivent aussi être réduits via entre autres à la mise à disposition des matériels et drogues d'urgence ainsi qu'une meilleure organisation des blocs opératoires.

L'évaluation des pertes sanguines peut se faire grâce à la pesée des pagnes. En effet, les protections mises sur les tables d'accouchements sont des pagnes (tissus africains) apportés par les patientes. Ainsi, afin de quantifier les pertes, les sages-femmes et/ou gardes-malades peuvent peser le pagne avant et après l'accouchement. Il en sera de même dans le post-partum immédiat où là encore, la garniture de protection est faite avec un pagne. Il suffira de le peser pour évaluer la perte sanguine de la patiente avant qu'elle n'aille dans le service des suites de couches.

Enfin, nous pourrons songer à la mise en place de feuilles de surveillance du post-partum en cas d' HPPI afin qu'il y ait une traçabilité des actes réalisés.

\section{Le rôle de la sage-femme}

La sage-femme joue un rôle important dans la prévention, le diagnostic et la prise en charge de l'HPPI. C'est elle qui suit la majorité des patientes durant leur grossesse, qui réalise la totalité ou 
presque des accouchements voie basse, et est en charge de la surveillance du post-partum immédiat. C'est aussi elle qui accueille les patientes transférées. Il est donc nécessaire que les sages-femmes africaines aient de bonnes connaissances théoriques et pratiques sur les conduites à tenir sur la prévention et le traitement d'une HPPI.

Ainsi, afin d'atteindre le cinquième objectif du millénaire pour le développement (OMD) de 2015 (31), qui vise à réduire de 75\% la mortalité maternelle entre 1990 et 2015, de nombreuses actions ont été mises en œuvre par différents organismes non gouvernementaux.

La déclaration commune de l'ASFB (Association des sages-femmes du Bénin) et de la SGOBT (Société de Gynécologie et d'Obstétrique du Bénin et du Togo) du 15 mai 2008 affirme «qu'il est impératif de compter des prestataires qualifiés et des services obstétricaux améliorés comme éléments centraux aux efforts visant la réduction de la mortalité maternelle » (32). Ainsi, l'action principale des organismes de santé est la promotion de la formation du personnel soignant sur place, en particulier les sages-femmes. En effet, d'après l'ONU, «la présence d'un personnel qualifié (médecin, infirmière ou sage-femme) est un facteur de diminution du nombre de décès » (2) C'est donc dans cette optique que l'AMREF (Association pour la Médecine et la Recherche en Afrique) a lancé en 2011, la campagne «stand up for african mothers ». Cette campagne a pour objectif de former et de remettre à niveau 15000 sages-femmes en Afrique sub-saharienne d'ici 2015, ce qui permettrait de réduire de $25 \%$ la mortalité maternelle. En 3ans, plus de 5000 sagesfemmes ont été formées dans six pays essentiellement en Afrique de l'Est. En 2014, la campagne s'ouvre en Afrique de l'ouest avec un premier programme lancé au Sénégal (33). D'autres associations comme l'association Gynécologie sans frontières (GSF), proposent aux sages-femmes françaises des missions humanitaires en Afrique, afin de partager leurs connaissances de la prise en charge des patientes avec leurs consœurs africaines. Ainsi en janvier 2014, une mission d'aide à 1'hôpital Saint Jean de Dieu à Afagnan (Togo).

Une sage-femme ougandaise, Esther Madudu, soutenue et formée par l'AMREF est candidate au prix Nobel de la paix de 2015. Le but étant de faire prendre conscience à l'ensemble de l'opinion international de la nécessité de l'amélioration de la santé maternelle dans les pays en développement. 


\section{Conclusion générale}

Les résultats de l'étude ont montré une prévalence d'HPPI de 4.7\%. Conformément aux recommandations de l'OMS, la réalisation de la GATPA, et pratiquée par les médecins et sagesfemmes au CHU-SO dans 95.6\% des accouchements. En ce qui concerne la prévention pour les patientes ayant un risque accru d'HPPI, le protocole observé associant ocytocine et misoprostol n'est pas conforme aux recommandations. Concernant la prise en charge de l'HPPI, les connaissances divergent entre les sages-femmes et les médecins en liens avec les compétences mobilisées. En effet, aucune sage-femme n'a mentionné la prise en charge chirurgicale en cas d'HPPI. L'ergométrine, encore administrée aux parturientes togolaises en cas d'HPPI ne doit plus faire parti de l'arsenal thérapeutique.

Cette étude a également montré que $85.7 \%$ des patientes pour qui une HPPI avait été diagnostiqué, n'étaient pas suivies au centre d'étude. Parmi elles, $66.7 \%$ ont été transférées au CHU pour HPPI. La cause principale de l'HPPI était la rétention placentaire. Il peut être proposé de développer la formation médicale continue des professionnels travaillant dans les centres de soins primaires (par exemple, démonstration de révision utérine sur simulateur). Les indications et l'utilisation des moyens médicamenteux dans la prise en charge de l'HPPI n'étaient pas optimales.

Enfin, nous avons aussi mis en évidence une sous-estimation importante des pertes sanguines dans le post-partum immédiat. Une pesée des pagnes, servant habituellement au recueil, avant et après l'accouchement pourrait permettre une estimation plus conforme à la réalité.

Le tamponnement intra-utérin par condom cathéter permettrait une prise en charge non médicamenteuse de l'HPPI. Le tamponnement intra-utérin pourrait ainsi constituer une alternative à l'utilisation du Méthergin ${ }^{\circledR}$.

La réalisation de ce travail de recherche a été l'occasion de sensibiliser la future professionnelle au domaine de la santé publique. Il me tarde à présent de m'engager, sous réserve de l'obtention de mon diplôme d'Etat de Sage-femme, dans des missions humanitaires en Afrique. 


\section{ANNEXES :}

\section{ANNEXE I : Carte $\boldsymbol{d} \boldsymbol{u}$ ToGo}

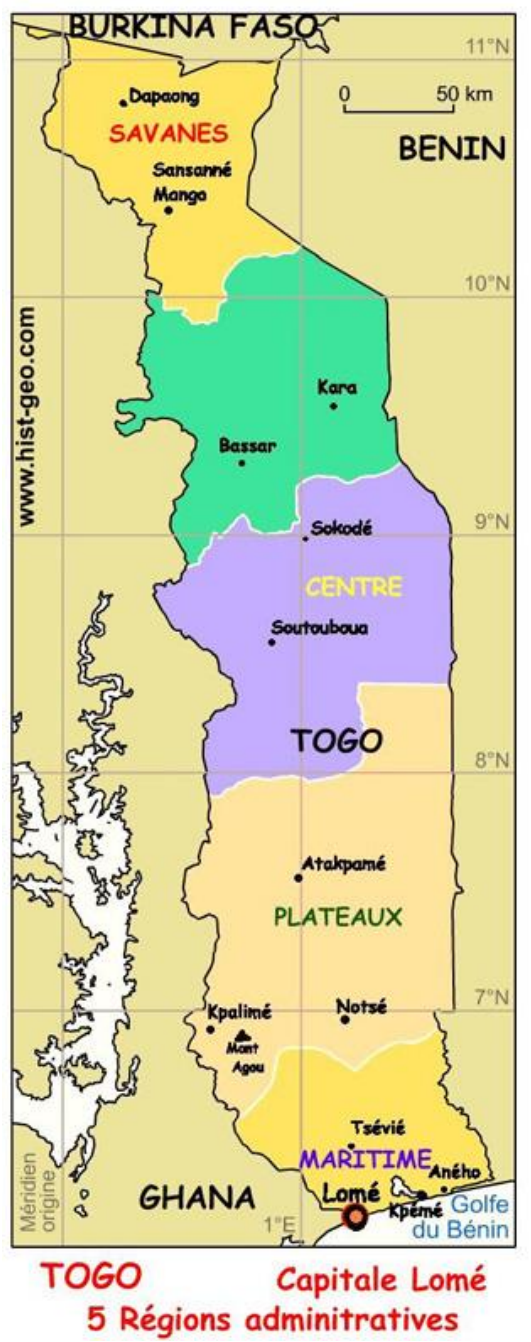

[Disponible sur www.google.fr] 
ANNEXE II : La Gestion Active de la Troisième Phase de l'Accouchement 


\section{ANNEXE III : Grille de remboursement des frais de santé par l'INAM}

(Institut National d'Assurance Maladie)

\begin{tabular}{|c|c|}
\hline \multicolumn{2}{|c|}{ PRISE EN CHARGE SOCIALE } \\
\multicolumn{2}{|c|}{ DES DEPENSES DE SANTE PAR L'INAM } \\
\hline Consultations générales & $80 \%$ \\
\hline $\begin{array}{c}\text { Consultation au centre de santé public pour les } \\
\text { enfants de moins de 5 ans }\end{array}$ & $100 \%$ \\
\hline Consultation de spécialité & $80 \%$ \\
\hline Frais de séjours hospitaliers & $90 \%$ \\
\hline Examen de laboratoire & $80 \%$ \\
\hline Intervention chirurgicale & $90 \%$ \\
\hline Petite chirurgie & $80 \%$ \\
\hline Soins infirmiers & $80 \%$ \\
\hline Accouchement & $100 \%$ \\
\hline Césarienne & $100 \%$ \\
\hline Soins ophtalmologies & $80 \%$ \\
\hline Soins dentaires & $80 \%$ \\
\hline Poche de sang et médicaments & $80 \%$ \\
\hline Echographie et radiologie & $80 \%$ \\
\hline
\end{tabular}


ANNEXE IV : Recommandations pour la pratique clinique (RPC) des soins obstétricaux et néonataux d'urgence en Afrique dans le cas de l'HPPI (www.sante.gov.ml) 


\section{GRILLE DE RECUEILS DES DONNEES SUR HEMORRAGIE DU POST-PARTUM IMMEDIAT}

\section{Gritères de la patiente:}

Age :

Taille :

Poids (à l'accouchement) :

Patiente suivie au CHU :
1. Oui 2. Non
Si non : 1. Patiente non suivie
2. Patiente référée Motif :

Ötutécédents médiccaux/gynécologiques/obstétricaux

\section{Gestité :}

\section{Parité :}

Antécédents de fausses-couches / IVG /MFIU
1. Fausse-couche
2. IVG
3. MFIU
4. NSP

Utérus cicatriciel :
1. Oui
2. Non
Si oui, combien et si possible pour quelle(s) raison(s) ?
Définition d'utérus cicatriciel : utérus ayant une cicatrice => césarienne, myomectomie, grossesse extra-utérine, rupture utérine, perforation utérine

\section{Antécédents HPP}
1. Oui
2. Non
3. NSP
Si oui, précisez (HPPI, HPP des 24h, traitements)

Troubles de l'hémostase (thrombopénie, hémophilie, maladie de willebrand...)
1. Oui
2. Non
3. NSP

\section{Grossesse actuelle}

Nombre de fœtus :
1. Singleton
2. 2
3. 3 
Suivi de grossesse :
1. Non
2. Peu de suivi
3. Suivi régulier (Au moins 4 CPN)

Placenta prævia :
1. Oui
2. Non

Grossesse pathologique :
1. Oui
2. Non
3. NSP

Si oui, précisez pathologie

\section{Déroulement du travaí:}

Terme :

Entrée en travail :
1. Spontané
2. Déclenchement
3. NSP

Déclenchement du travail :

$\begin{array}{llll}\text { 1. Pour cause fœtale } & \text { 2. Pathologie maternelle } & \text { 3. Terme dépassé } & \text { 4.NSP }\end{array}$ Précisez la cause Utérotonique utilisé pour le déclenchement :
1. Cytotec
2. Ocytocine
3. NSP

Si travail spontané, utilisation d'ocytocine pendant le travail:

1. Oui

3. Non

Si oui, dose reçue

Anesthésie :

1. Aucune

2. Rachianesthésie

3. $A G$

4.NSP

Durée du travail :

\section{Tocouchement.}

Heure de l'accouchement :

Présentation fœtale :
1. Sommet
2. Siège
3. Transverse
4. NSP

Extraction fœtale instrumentale par voie basse:
1. Oui
2. Non

Si oui

1. Forceps 2. Ventouse

Manœuvres obstétricales pratiquées :
1. Oui
2. Non

Si oui, quelle(s) manœuvre(s)

\section{Lésions périnéales :}

$\begin{array}{llll}\text { 1. Périnée intacte } & \text { 2. Episiotomie } & \text { 3. Déchirure (degré ............) 4. NSP }\end{array}$ Lésions internes :
1. Déchirure col
2. Vagin
3. NSP

Extraction fœtale par voie haute :
1. Césarienne programmée
2. Césarienne en urgence avant travail

2. Césarienne en urgence pendant travail

Indications de la césarienne 


\section{GoYTP:}

Ocytocique immédiatement après l'expulsion fœtale :
1. Oui
2. Non

Combien d'unités?

Massage utérin :
1. Oui
2. Non
3. NSP

Traction contrôlée du cordon :
1. Oui
2. Non
3. NSP

\section{Pracenta:}

Délivrance :
1. Délivrance assistée
2. Naturelle
3. Artificielle
4. Manuelle
5. Complète
7. Incomplète

Examen du placenta :
1. Oui
2. Non
3. NSP

\section{Pouveau-né(s):}

Poids à la naissance :

J1 :

J2:

J3 :

\section{Survoillance dus post-partum imomédiat.}

Durée de la surveillance en salle (de naissance / réveil) : Eléments de surveillance :
1. Globe utérin :
oui
non
NSP
2. Saignements :
oui
non
NSP
3. Pouls:
oui
non
NSP
4. TA :
oui
non
NSP
5. $\mathrm{T}^{\circ}$ :
oui
non
NSP

Fréquence de la surveillance pendant la durée de la surveillance:

1. 1 fois

2. 2 fois

3.Au moins 3 fois

4.NSP

Personnel(s) en charge de la surveillance du PP

1. Sage-femme oui non

2. IDE

oui

non

3. étudiante

oui

non

4. Autres précisez 
Utilisation d'utéro tonique pendant le PP :

1. Oui

Si oui

4. Ocytocine
2. Non

5. misoprostol
3. NSP

6. Méthergin

Examen médical autorisant la sortie de salle de naissance / salle de réveil:
1. Oui
2. Non
3. NSP

Mode de sortie de salle de naissance / salle de réveil:

$\begin{array}{ll}\text { 1. SDC 2. Transfert en réanimation } & \text { 2. }\end{array}$

3. Décès

4.Domicile 5. autre 
Quantification pertes sanguines lors du diagnostic :
1. Oui
2. Non
3. NSP

Si oui
1. $<500 \mathrm{cc}$
2. De 500 à $1000 c c$
3. De 1000 à 1500
4. $>1500 c c$

Professionnels sur place en cas d'HPP :
1. $\mathrm{SF}$
Oui
Non
2. CDG
Oui
Non
3. IDG
Oui
Non
4. Anesthésiste
Oui
Non
5. IDE
Oui
Non

Causes probables de I'HDD :

1. Atonie utérine Oui Non

2. Plaie filière génitale Oui Non

3. Déchirure cervicale Oui Non

4. Rupture utérine Oui Non

5. Episiotomie hémorragique Oui Non

6. HRP Oui Non

7. Rétention placentaire Oui Non

PEC de I'HDD

1. RU Oui Non

NSP

2. Massage utérin Oui Non

NSP

3. $S A D / A-R \quad O u$

Non NSP

4. Pose VVP Oui Non

NSP

5. Examen de la filière génitale oui non NSP

6. Prélèvement sanguins Oui Non NSP

7. Utilisation d'utéro toniques Oui Non NSP

précisez quel(s) utéro tonique(s) : ocytocine -misoprostol - méthergin........

Quantité totale administrée à la patiente

8. Transfusion Oui Non

NSP

Si oui, précisez quantité et type de PSL (CG, Fg, plaquettes...).......

9. ligature vasculaire Oui Non

10. Hystérectomie d'hémostase Oui Non

\section{Organisation des noins}

Heure de diagnostic de l'HDD :

Heure d'arrivée de l'équipe complète :

Heure début des traitements :

Heure de fin :

Tolérance maternelle :

Score de Glasgow* : $\quad$ /15 
Examen médical autorisant la sortie de salle de naissance / salle de réveil:

2. Oui

2. Non

Mode de sortie de salle de naissance /salle de réveil:

2. SDC

2. Transfert en réanimation

4. Domicile

5. autre
3. NSP

3. Décès

\section{*Score de Glasgow :}

\$) Ouverture des yeux.

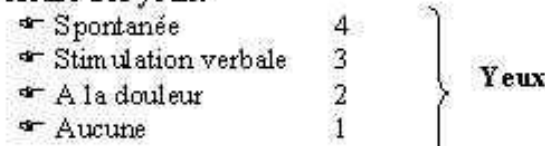

6 Réponse verb ale.

$$
\left.\begin{array}{ll}
\approx \text { Orientée } & 5 \\
\approx \text { Confuse } & 4 \\
\approx \text { Inapropriée } & 3 \\
\approx \text { Incompréhensible } & 2 \\
- \text { Aucune } & 1
\end{array}\right\} \text { Verbal }
$$

t8 Réponse mo trice.

- Obéissance aux ordres 6

* Flexion adaptée

- Flexion non adaptée 4

- Décortication 3

- Décérébration 2

$\approx$ Aucune

Moteur 


\section{ENTRETIEN AVEC SAGE-FEMME / OBSTETRICIEN}

\section{Objectifs : Evaluer la formation du personnel dans le cadre de la gestion d'une HPPI}

\section{Durée : 20minutes}

\section{Totivité professionnelle:}

1. Depuis combien de temps exercez-vous en tant que professionnel de l'obstétrique ?
$<3 a n s$
3-6 ans
$>7$ ans

\section{Formation théorique suivie:}

2. Quelles sont au Togo les critères pour exercer en tant que sage-femme ? (diplôme d'état ou selon nombre d'années d'expérience) ou obstétricien ?

- Quelle est la durée de votre formation ?

- Quel est le contenu de la formation reçue ? (Matières étudiées)

- Avez-vous fait vos études de sage-femme / obstétricien au Togo ou à l'étranger ? Si à l'étranger, dans quel pays?

3. Quelles sont les compétences de la SF / obstétricien en salle de naissance ?

- A partir de quel moment faites-vous appel au gynécologue-obstétricien de garde ?

- A partir de quel moment êtes-vous appelés en salle de naissance pour une HPP ?

4. Quel est le rôle de la sage-femme / obstétricien en salle de naissance ? 


\section{Gonnarissances:}

6. D'après vous, qu'est-ce qu'une hémorragie du post-partum immédiat ?

- Définition d' « immédiate »(30minutes ? 3h ?...)

- La quantité des pertes sanguines est-elle la même pour l'accouchement voie basse et la césarienne?

7. Quelles peuvent en être les causes ?

8. Imaginons que vous ayez à assurer le suivi de grossesse d'une patiente présentant un FDR d'HPP (tel un ATCD d'HPP, placenta prævia...), quel (s) moyens de prévention mettriezvous en place pour éviter l'HPP à l'accouchement?

9. Avez-vous déjà entendu parler de la GATPA ?

- Si oui, en quoi consiste-t-elle?

- Appliquez-vous ces recommandations ? Si non, pourquoi ?

\section{Giestion de / FPPD:}

10. Avez-vous déjà eu à gérer des HPP ?

- Si oui, Combien ?

- Quel en fut l'issue pour les patientes?

- Si décès de la patiente, selon vous quelle en a été la cause ? (une PEC inadaptée, un diagnostic tardif ou autres)

11. En tant que sage-femme / obstétricien quel est votre rôle dans la gestion de l'HPP ?

- Quelles sont les actes que vous pratiquez en tant que SF en attendant l'obstétricien ?

- En tant qu'obstétricien, qu'attendez-vous de la sage-femme en cas d'HPP ?

12. La PEC de l'HPP est-elle protocolaire ou est-elle professionnel dépendant ?

- (Si elle est professionnelle dépendante, quelle est votre PEC personnelle ?) 
13. Afin de mieux comprendre l'organisation du service, pourriez-vous me décrire la PEC type d'une HPP au sein de cette maternité?

- Quels sont les membres du personnel sur place?

- Quels sont les traitements ? La posologie?

\section{Kessentipersonnel:}

14. Selon vous quelle(s) peut (peuvent) être la (es) cause (s) du taux élevés d'HPP en Afrique ?

15. Selon vous quelles sont les limites à une PEC optimale de I'HPP ?

16. Pensez-vous que l'on doit accroître la prévention ?

- Si oui, de quelle façon ?

Merci de votre disponibilité 


\section{$\underline{\text { Références bibliographiques }}$}

1. INED. La mortalité maternelle dans le monde. [Internet]. [Consulté le 20.10. 2013]

Disponible sur internet:

$<$ http://www.ined.fr/fr/tout_savoir_population/fiches_pedagogiques/duree_de_vie_deces_

mortalite/mortalite_maternelle_monde_ >

2. ONU. Objectifs du Millénaire pour le développement, objectif 5: améliorer la santé maternelle [Internet]. [Consulté le 25.02.2014]

Disponible sur: < https://www.un.org/fr/millenniumgoals/maternal.shtml>

3. L'hémorragie du postpartum: un défi pour une maternité sans risques. [Internet].

[Consulté le 20.10.2013]. Disponible sur:

$<$ http://gynuity.org/downloads/factsht_challengesafemotherhood_fr.pdf ] $>$.

4. TOGO - statistiques-mondiales.com - Statistiques et carte. [Internet]. Consulté le 20.10.2013].Disponible sur:< http://www.statistiques-mondiales.com/togo.htm $>$

5. AGBOBLI Abla. Le suivi de la grossesse, de l'accouchement et de la prise en charge des nouveau-nés dans deux maternités à Lomé, au Togo en 2008. Mémoire/ Ecole de sagesfemmes : faculté Henri Poincaré (Nancy 1) ; 2009

6. Kramer MS, C B, Abenhaim H, Dahhou M, Rouleau J, Mehrabadi A, et al. Incidence, risk factors, and temporal trends in severe postpartum hemorrhage. American Journal of obstetrics and gynecology; 2013; 209(5):449.e1-7.

7. Wetta LA., Szychowski JM, Seals S., Mancuso MS., Biggio JR., Tita AT. Risk factors for uterine atony/postpartum hemorrhage requiring treatment after vaginal delivery. American journal of obstetrics and gynecology. 2013; 209(1):51.e1-51.e6.

8. Lao TT, Sahota DS., Cheng YK., Law LW., Leung TY. Advanced maternal age and postpartum hemorrhage - risk factor or red herring? Journal of Maternal-fetal and Neonatal Medicine; 2013.

9. Second-trimester placental location and postpartum hemorrhage. Journal of Ultrasound in Medicine. 2013; 32(4):631-6.

10. Confédération internationale des sages-femmes (ICM) et Fédération internationale de gynécologie et d'obstétrique (FIGO). Prise en charge du troisième stade du travail pour prévenir l'hémorragie du post-partum. Journal of obstetrics and gynecology Canada. $2003 ; 25: 954-955$.

11. Soltani H. Massage utérin dans la prévention de l'hémorragie du post-partum. Bibliothèque de Santé Génésique de l'OMS; Genève: Organisation mondiale de la Santé. 
12. Initiative pour la prévention de l'Hémorragie du Post-partum (POPPHI). La prévention de l'hémorragie du post-partum: la gestion active de la troisième période de l'accouchement. Formation des accoucheurs qualifiés. [Internet]. 2008. Disponible sur: http://www.pphprevention.org/files/GATPA Guidedufacilitateur 002.pdf [consulté le 16.09.2013]

13. WJ $P$, Elbourne $D, M c D o n a l d ~ S$. Active versus expectant management in the third stage of labour. Cochrane Database of Sytematic Reviews. 2000;3(CD00007).

14. Utilisation du syntocinon commission de la transparence [Internet] Haute Autorité de Santé (HAS). Disponible sur: http://www.sigma-tau.fr/media/pdf/syntocinon has.pdf [Consulté le 20.10.2013]

15. Consignes d'utilisation du misoprostol [Internet]. > [Consulté le 16.10.2013]

Disponible sur : <http://www.misoprostol.org/File/Other_G_Gynuity_PPH_guidelines_Fre.pdf>

16. Mobeen N., Durocher J., Zuberi NF., Jahan N, Blum J., Wasim S., et al. Administration of misoprostol by trained traditional birth attendants to prevent postpartum haemorrhage in homebirths in Pakistan: a randomised placebo-controlled trial. BJOG: An international journal of obstetrics \& gynecology. 2011; 118(3):353-61.

17. Lokugamage AV, Sullivan KR, Niculescu L, Tigere $P$, et al. A randomised study comparing rectally administered misoprostol versus syntometrine combined with an oxytocin infusion for the cessation of primary postpartum haemorrhage. Acta obstetrica gynecologica scandinavica 2001;80 (9):.835-839.

18. Gülmezoglu AM., Forna F., Villar J., Hofmeyr GJ. Prostaglandins for prevention of postpartum hemorrhage [Internet]. 2007. [Consulté le 18.10.2013] Disponible sur: <http://www.ncbi.nlm.nih.gov/pubmed/17636640>.

19. McDonald S, Abbott JM, Higgins SP. Prophylactic ergométrine-oxytocin versus oxytocin for the third stage of labour [Internet]. The Cochrane Library; 2007. Disponible sur: http://apps.who.int/rhl/reviews/CD000201.pdf [Consulté le 18.10.2013].

20. Dougas M. Donner aux populations vulnérables de l'Afrique subsaharienne un accès aux soins obstétricaux: stratégie avancée de consultations prénatales. Médecine tropicale. 2011;71:526-8.

21. Baxerres C, F D, Gbofan F., Le Hesran JY. Pratique des femmes et recommandations biomédicales en matière de grossesse et d'accouchement au Sénégal et au Bénin. Médecine tropicale. 2011; 71(4):388.

22. N'dinga HG, GR O, Angouono-Moke, lloki LH. Accouchement sans assistance médicale à I'hôpital de base de Talagaï à Brazzaville (Congo). Médecine tropicale. 2011;71(3):278-80.

23. Pambou O., Ekoundzola JR., Yoca G., Uzan S. Les hémorragies graves de la délivrance au C.H.U. de Brazzaville [Internet]. Disponible sur :

http://www.santetropicale.com/resume/74305.pdf [consulté le 15.10. 2013] 
24. Ghana Health services-ministry of Healthy. Prevention and treatment of Post-partum haemorrhage [Internet]. [consulté le10.10.2013].

Disponible sur: http://www.knov.nl/docs/uploads/Ghana joint statement - Eng.pdf

25. Bienvenue sur INAM TOGO le site Internet de I'Institut National d'Assurance Maladie du TOGO [Internet]. [consulté le 20.10.2013].Disponible sur: <http://www.inam.tg/ >

26. Sant'anna M., C M, Rapp. C. Accessibilité des femmes à la surveillance biologique des grossesses au Togo. Médecine tropicale. 2011;71(4):389.

27. APKPADZA K., Baeta $S A$. Hodonou KS. Les hémorragies de la délivrance à la clinique de gynécologie-obstétrique du CHU Tokoin-Lomé (Togo) de 1988 à 1992. Médecine d'Afrique Noire. 1994;41(11):601-603.

28. Adama-hondegla A., Aboubakari A-S., Agbekponou K., Bassowa A., Agbetra N., Akpadza K. Evolution de la mortalité maternelle au C.H.U. Tokoin de Lomé de 2005 à 2007 (3ans) et de 1987 à 2007 (20ans) [Internet]. [consulté le 15.03.2013] Disponible sur: http://sago.sante.gov.ml/pdf2008/EVOLUTION\%20DE\%20LA\%20MORTALITE\%20MATERN ELLE\%20AU\%20CHU\%20TOKOIN\%20DE\%20LOME\%20DE\%202005\%20A\%202007\%20\%28 3\%20ans\%29\%20ET\%20DE\%201987\%20A\%202007\%20\%2820\%20ans\%29.pdf

29. Belghiti et coll., Oxytocin during labour and risk of severe postpartum haemorrhage: a population-based, cohort-nested case-control study, BMJ Open 2011

30. Campagne pour l'accélération de la réduction de la mortalité maternelle en Afrique [Internet] [Consulté le 10.02.2014]. Disponible sur internet $<$ http://www.afro.who.int/en/togo/press-materials/item/4568-le-togo-\%C3\%A9value-lamise-en-oeuvre-de-son-programme-de-subvention-de-la-c\%C3\%A9sarienne.html >

31. Association des sages-femmes du Bénin (ASFB) et Société de gynécologie et d'obstétrique du Bénin et du Togo (SGOBT). Prévention et traitement de l'hémorragie du post-partum, 15 mai 2008

32. AMREF < https://www.amref.fr $>$ [Consulté le 22.02.2014]

33. USAID hémorragie du postpartum : prévention et prise en charge [Internet] [Consulté le 21.02.2014] Disponible sur <http://www.k4health.org/sites/default/files/PPH\%20Tec\%20Brief_French.pdf> 


\section{Glossaire des abréviations :}

CEDEAO : Communauté Economique Des Etats de l'Afrique de l'Ouest

C.H.U : Centre Hospitalier Universitaire

CPS : Comprimés

CMS : Centre Médico-social ou Centre Médical de Santé

DTT : Durée Totale du Travail

FCFA : Franc CFA ( $1 €=655$ FCFA)

FIGO: Fédération International de Gynécologie et d'Obstétrique

GATPA : Gestion Active de la Troisième Phase de l'Accouchement

HPP(I) : Hémorragie du Post-partum (Immédiat)

ICM : International Confederation of Midwives( Confédération international des sagesfemmes)

ML : Millilitres

NN : Nouveau-né

OMS : Organisation Mondiale de la Santé

PSL : Produits Sanguins Labiles

RU : Révision utérine

SAGO : Société Africaine de Gynécologie- Obstétrique

TH : Taux d'Hémoglobine

VVP : Voie Veineuse Périphérique 
